\title{
A POISSON-PLANCHEREL FORMULA FOR THE UNIVERSAL COVERING GROUP WITH LIE ALGEBRA OF TYPE $B_{n}$
}

\author{
PETER DOURMASHKIN
}

\begin{abstract}
A proof is given for the Poisson-Plancherel formula for Lie groups of type $B_{n}$ using the recurrence relations for the Plancherel function on adjacent Cartan subalgebras given in [12] and the recurrence relations for the discrete series constants which determine a $G$-invariant generalized function on $\mathbf{g}^{*}$ appearing in the formula.
\end{abstract}

\section{INTRODUCTION}

Let $G$ be a general Lie group and let $\hat{G}$ denote the set of isomorphism classes of irreducible unitary representations of $G$. The theory of harmonic analysis on $G$ has been the focus of a considerable amount of study. An "abstract" Plancherel formula (see [3]) has existed for a long time. The "explicit" Plancherel formula has now been extended to a broad class of Lie groups [1, 2, $5,8,10$, and 11].

For any real Lie group, Vergne [16] conjectured a Poisson-Plancherel formula which generalizes the Poisson summation formula. This conjecture has been proved by Duflo [4] when $G$ is complex algebraic, by Vergne [16] when $G$ is linear semisimple, and by Torasso [15] when $G$ is of Takiff type.

The motivation for the conjecture can best be illustrated by the following example.

Let $G=\mathfrak{R} / \mathbf{Z}$ be the torus. The Lie algebra $\mathrm{g}$ of $G$ is $\mathfrak{R}$. The map exp: $\mathfrak{R} \rightarrow \mathfrak{R} / \mathbf{Z}$ mapping $\mathbf{g}$ onto $G$ is the natural quotient map. Let 1 be the identity element in $G$. Let $\mathbf{g}_{G}=\{X \in \mathbf{g} \mid \exp X=1\} \simeq \mathbf{Z}$. Let $\mathbf{g}^{*}$ be the dual of $\mathbf{g}$. Hence $\mathbf{g}^{*} \simeq \mathfrak{R}$. Let

$$
\mathbf{g}_{G}^{*}=\left\{f \in \mathbf{g}^{*} \mid(f, X) \in 2 \pi \mathbf{Z}, X \in \mathbf{g}_{G}\right\}
$$

be the dual lattice to $\mathbf{g}_{G}$. Then $\mathbf{g}_{G}^{*}$ is canonically isomorphic to $\hat{G}$.

Let $\phi \in \mathscr{S}(\mathbf{g})$. Then the Poisson summation formula is given by

$$
\sum_{n \in \mathbf{g}_{G}} \phi(n)=\sum_{f \in \mathbf{B}_{G}^{*}} \hat{\phi}(f),
$$

where $\hat{\phi}$ is the Fourier transform of $\phi$.

Received by the editors June 12, 1986 and, in revised form, November 18, 1987.

1980 Mathematics Subject Classification (1985 Revision). Primary 22E46; Secondary 17B15. 
We note that the Poisson summation formula shows that there exists a $G$ invariant tempered distribution, the $\delta$-distribution of the lattice $\mathbf{g}_{G}$, whose Fourier transform is the $\delta$-function of the discrete set $\mathbf{g}_{G}^{*} \simeq \hat{G}$.

This paper proves a modified version of the conjecture when $G$ is the universal covering group with Lie algebra $\mathbf{g}=\operatorname{so}(p, q ; \mathfrak{R}), p+q=2 n+1, p<q$, and $p$ or $q \neq 0,1,2$.

Fix a character $\chi$ of the center $Z$ of $G$. Let

$$
\mathbf{g}_{\mathbf{C}}^{Z}=\left\{X \in \mathbf{g}_{\mathbf{C}} \mid \exp (\operatorname{ad} X)=1\right\} \text {. }
$$

Let $\mathbf{g}^{Z}=\mathbf{g}_{\mathbf{C}}^{Z} \cap \mathbf{g}$. Let $t$ be the maximal compact Cartan subalgebra of $\mathbf{g}$. Let $\Delta\left(\mathbf{g}_{\mathbf{C}}, t_{\mathbf{C}}\right)$ be the roots of $\mathbf{t}_{\mathbf{C}}$ in $\mathbf{g}_{\mathbf{C}}$. Let $\Delta^{+}$be a choice of positive roots. For each $\alpha \in \Delta^{+}$, let $H_{\alpha}$ be the associated coroot. Let $\pi_{\mathrm{t}}=\prod_{\alpha \in \Delta^{+}} H_{\alpha}$. Let $\mathbf{t}^{Z}=\mathbf{g}^{Z} \cap \mathbf{t}$.

Define a tempered distribution for $\phi \in \mathscr{S}(\mathbf{g})$,

$$
\phi \mapsto \sum_{\xi \in \mathbf{t}^{Z}} \chi(\exp \xi)\left(D_{\pi_{\mathrm{t}}} M \phi\right)(\xi),
$$

where $D_{\pi_{t}}$ is the constant coefficient differentiation operator associated to $\pi_{t}$, and $M$ is the invariant integral of Harish-Chandra [6].

Results of Harish-Chandra [6] and Rossmann [13] provide a Fourier inversion formula for this sum of distributions:

$$
\sum_{\xi \in \mathbf{t}^{Z}} \chi(\exp \xi)\left(D_{\pi_{\mathbf{t}}} M \phi\right)(\xi)=\int_{\mathbf{g}^{*}} V^{\chi}(f) \hat{\phi}(f) d f,
$$

where $V^{\chi}(f)$ is a $G$-invariant generalized function on $\mathbf{g}^{*}$, which is determined by recurrence relations on adjacent Cartan subalgebras.

Introduce the following notation. Let $\mathbf{g}=\mathbf{k} \oplus \mathbf{p}$ be the Cartan decomposition of $\mathbf{g}$ with Cartan involution $\theta$. Let $\mathbf{h}$ be any $\theta$-stable Cartan subalgebra of $\mathbf{g}$. Then $\mathbf{h}=\mathbf{h}_{k} \oplus \mathbf{h}_{p}$, where $\mathbf{h}_{k}=\mathbf{h} \cap \mathbf{k}$ and $\mathbf{h}_{p}=\mathbf{h} \cap \mathbf{p}$. Let $\mathbf{h}_{\mathbf{C}}^{Z}=$ $\mathbf{h}_{\mathbf{C}} \cap \mathbf{g}_{\mathbf{C}}^{Z}$. Let $\Phi=\Phi\left(\mathbf{g}_{\mathbf{C}}, \mathbf{h}_{\mathbf{C}}\right)$ be the root system. Let $\Phi^{+}$be a choice of positive roots. Let $\Phi_{\mathfrak{R}}^{+}$be the real positive roots. For each $\alpha \in \Phi_{\mathfrak{R}}^{+}$, let $H_{\alpha}$ be the associated coroot. Let $C_{\mathfrak{R}}^{+}=\left\{f \in \mathbf{h}^{*} \mid\left(H_{\alpha}, f\right)>0, \alpha \in \Phi_{\mathfrak{R}}^{+}\right\}$. Let $W\left(\mathbf{t}_{\mathbf{C}}, \mathbf{h}_{\mathbf{C}}\right)=$ $\left\{g \in G_{\mathbf{C}} \mid g\left(\mathbf{t}_{\mathbf{C}}\right)=\mathbf{h}_{\mathbf{C}}\right\}$.

The generalized function $V^{\chi}(f)$ is completely determined by its restriction to all the subspaces $\mathbf{h}^{*}$. Then for $\lambda \in C_{\mathfrak{R}}^{+}$,

$$
V_{\mathbf{h}}^{\chi}(\lambda)=\sum_{\xi \in \mathbf{h}_{\mathbf{C}}^{Z}} r_{\mathbf{h}}^{\chi}\left(\xi, \Phi_{\mathfrak{R}}^{+}\right) e^{-i(\xi, \lambda)},
$$

where

$$
r_{\mathbf{h}}^{\chi}\left(\xi, \Phi_{\mathfrak{R}}^{+}\right)=\sum_{w \in W\left(\mathbf{t}_{\mathbf{C}}, \mathbf{h}_{\mathrm{C}}\right)} \chi\left(\exp w^{-1} \xi\right) h\left(w ; \Phi_{\mathfrak{R}}^{+} ; w^{-1} \xi\right) .
$$

The $h\left(w ; \Phi_{\mathfrak{R}}^{+} ; \xi\right)$ are a set of constants related to the discrete series constants defined by Harish-Chandra [7] which are completely determined by an inductive argument on the rank of $\Phi$. 
Let $H=H_{K} \cdot H_{p}$ be the Cartan subgroup of $G$ associated with $\mathbf{h}$. Let $H^{0}$ be the connected component of $H$. Let $\hat{H}(\chi)=\{\tau \in \hat{H} \mid \tau(z)=\chi(z) \cdot$ Id, $z \in Z\}$. For $\tau \in \hat{H}(\chi)$, define $b \in \hat{H}_{K}, \nu \in \mathbf{h}_{p}^{*}$ such that $\left.\tau\right|_{H_{K}}=b$ and $\tau(\exp X)=e^{i(\nu, X)}$ for $X \in \mathbf{h}_{p}$. For each $\alpha \in \Phi_{\Re}^{+}$, choose $X_{\alpha} \in \mathbf{g}_{\alpha}$ such that $\theta\left(X_{\alpha}\right)=-X_{-\alpha}\left[X_{\alpha}, X_{-\alpha}\right]=H_{\alpha}$, where $\alpha\left(H_{\alpha}\right)=2$. Let $\rho_{\mathfrak{R}}=\frac{1}{2} \sum_{\alpha \in \Phi_{\mathfrak{R}}^{+}} \alpha$. Let $m_{\alpha}=\exp \left(\tau\left(X_{\alpha}+\theta\left(X_{\alpha}\right)\right)\right)$.

Define for $\tau \in \hat{H}(\chi)$,

$$
P(\tau)=\frac{1}{[\operatorname{dim} b]} \# \Phi_{\Re}^{+} \prod_{\alpha \in \Phi_{\Re}^{+}} \operatorname{tr}\left[\frac{\operatorname{sh}\left(\pi \nu, H_{\alpha}\right) \cdot \operatorname{Id}}{\operatorname{ch}\left(\pi \nu, H_{\alpha}\right) \cdot \operatorname{Id}+(-1)^{\left(\rho_{\mathfrak{R}}, H_{\alpha}\right)} \cdot \Lambda\left(b, m_{\alpha}\right)}\right],
$$

where $\Lambda\left(b, m_{\alpha}\right)=\frac{1}{2}\left(b\left(m_{\alpha}\right)+b\left(m_{\alpha}\right)^{-1}\right)$. Let $\mathbf{h}^{*}(\chi)=\left\{\lambda \in \mathbf{h}^{*} \mid \chi(\exp X)=\right.$ $\left.e^{i(\lambda, X)}, X \in \mathbf{h} \cap \mathbf{g}^{Z}\right\}$. For $\lambda \in C_{\mathfrak{R}}^{+} \cap \mathbf{h}^{*}(\chi)$, define

$$
P_{h}^{\chi}(\lambda)=\frac{1}{\left[H: H^{0} Z\right]} \sum_{\substack{\tau \in \hat{H}(\chi) \\ d \tau=i \lambda}}(\operatorname{dim} \tau)^{2} P(\tau)
$$

The function $P(\tau)$ is the Plancherel product function introduced by HarishChandra [8]. Extend $P_{\mathrm{h}}^{\chi}(\lambda)$ by $G$-invariance to a $G$-invariant function on $G$. $\mathbf{h}^{*}(\chi)$.

For $\lambda \in \mathbf{h}^{*}(\chi)$, define

$$
R_{\mathbf{h}}^{\chi}(\lambda)=\sum_{\delta \in \mathbf{h}^{Z}} e^{-i(\delta, \lambda)} \chi(\exp \delta)
$$

Then $R_{\mathrm{h}}^{\chi}(\lambda)$ extends to a $G$-invariant positive generalized function on $\mathbf{g}^{*}$ with support on the set of orbits of regular elements of $\mathbf{h}^{*}(\chi)$.

The modified version of the Poisson-Plancherel conjecture can be stated as follows.

Theorem (3.5). Let $G$ be the universal covering group with Lie algebra $\mathbf{g}=$ $\operatorname{so}(p, q ; \Re), p+q=2 n+1, p<q$, and $p$ or $q \neq 0,1,2$. Let $\chi$ be a fixed character of the center $Z$ of $G$. Then

$$
V_{\mathrm{h}}^{\chi}(\lambda)=R_{\mathrm{h}}^{\chi}(\lambda) P_{\mathrm{h}}^{\chi}(\lambda)
$$

for all $\boldsymbol{\theta}$-stable Cartan subalgebras $\mathbf{h}$ of $\mathbf{g}$.

The fact that there is an algebraic relation between the Plancherel function and the discrete series constants was suggested by the work of Herb [9].

The proof of this theorem will rely on a theorem of D. Feterson [12]. This theorem enables one to directly relate the Plancherel product function $P_{\mathbf{h}}^{\chi}(\lambda)$ to the constants $r_{h}^{\chi}(\lambda)$ appearing in the expansion of the $G$-invariant function $V_{h}^{\chi}(\lambda)$.

A brief outline of the contents of this paper is given below. 
In $\S 1$, all necessary definitions and notations are introduced. Characters on root lattices are introduced and critical lemmas which relate their values on adjacent Cartan subalgebras are proved.

In $\S 2$, a theorem of $D$. Peterson is introduced which describes the matching conditions which are satisfied by a function closely related to the Plancherel product function. Then the Plancherel function is described and expressed as a power series expansion over a root lattice with coefficients which satisfy the recurrence relations in the Peterson theorem. Proposition 2.4 enables one to identify the coefficients with constants related to the discrete series constants defined by Harish-Chandra.

In $\S 3$, the Poisson-Plancherel formula, Theorem 3.4 and Theorem 3.5, is proved.

The author would like to express his gratitude to his thesis advisor, Michele Vergne, for the inspiration and the many helpful discussions involved in this work.

In this section we describe the center $Z$ of $G$ and a fixed character $\chi$ on $Z$. We then introduce notation describing the adjacent Cartan subalgebras. We conclude with a series of lemmas describing character identities on lattices contained in $\mathbf{h}^{*}$.

Let $G$ be the universal covering group with Lie algebra $g=\operatorname{so}(p, q ; \Re)$, $p+q=2 n+1, p<q$, and $p$ or $q \neq 0,1,2$. Let $G_{1}=\operatorname{Spin}(p, q)$. The group $G_{1}$ is a real linear semisimple group with Lie algebra $\mathbf{g}$. Let $G_{a}=\operatorname{SO}(p, q)$. The group $G_{a}$ is the adjoint group of $G_{1}$ with Lie algebra $\mathbf{g}$. Let $\pi$ be the two-fold covering map from $G$ to $G_{1}$.

Let $K_{a}=\mathrm{SO}(p) \times \mathrm{SO}(q), K_{1}$, and $K=\operatorname{Spin}(p) \times \operatorname{Spin}(q)$ be the respective maximal compact subgroups for $G_{a}, G_{1}$, and $G$. Let $\pi_{p}\left(\pi_{q}\right)$ be the two-fold covering map from $\operatorname{Spin}(p) \quad(\operatorname{Spin}(q))$ to $\operatorname{SO}(p) \quad(\operatorname{SO}(q))$. Let $\left\{1, \varepsilon_{p}\right\}$ and $\left\{1, \varepsilon_{q}\right\}$ be the respective elements in $\operatorname{Spin}(p)$ and $\operatorname{Spin}(q)$ which project under $\pi_{p}$ and $\pi_{q}$ to the identity. Then $K_{1} \simeq \operatorname{Spin}(p) \times \operatorname{Spin}(q) / \varepsilon_{p} \sim \varepsilon_{q}$.

Let $Z\left(Z_{1}\right)$ be the center of $G\left(G_{1}\right)$. Then $Z \simeq \mathbf{Z} / 2 \mathbf{Z} \times \mathbf{Z} / 2 \mathbf{Z}=\left\{1, \varepsilon_{p}, \varepsilon_{q}\right.$, $\left.\varepsilon_{p} \cdot \varepsilon_{q}\right\}$ and $Z_{1} \simeq \mathbf{Z} / 2 \mathbf{Z}=\left\{1, \varepsilon_{p}=\varepsilon_{q}\right\}$, where $\pi$ sends $\left\{1, \varepsilon_{p} \cdot \varepsilon_{q}\right\}$ into 1 and $\left\{\varepsilon_{p}, \varepsilon_{q}\right\}$ into $\varepsilon_{p}$.

In the remainder of this paper, fix a character $\chi$ on the center $Z$ of $G$ such that

$$
\chi\left(\varepsilon_{p} \cdot \varepsilon_{q}\right)=-1 .
$$

Let $\mathbf{g}_{\mathbf{C}}^{Z}=\left\{X \in \mathbf{g}_{\mathbf{C}} \mid \exp (\operatorname{ad} X)=1\right\}$ and $\mathbf{g}^{Z}=\mathbf{g}_{\mathbf{C}}^{Z} \cap \mathbf{g}$. Then for $X \in \mathbf{g}^{Z}$, $\exp _{G}(X) \in Z$.

Let $\mathbf{g}=\mathbf{k} \oplus \mathbf{p}$ be the Cartan decomposition of $\mathbf{g}$ where $\theta$ is the corresponding Cartan involution. Let $\mathbf{h}$ be any $\theta$-stable Cartan subalgebra of $\mathbf{g}$. Then $\mathbf{h}=\mathbf{h}_{k} \oplus \mathbf{h}_{p}$, where $\mathbf{h}_{k}=\mathbf{h} \cap \mathbf{k}$ and $\mathbf{h}_{p}=\mathbf{h} \cap \mathbf{p}$. Let $H$ be the centralizer of 
h in $G . H$ is called the Cartan subgroup of $G$ associated to h. Let $H^{0}$ be the connected component of $H$. Let $H_{P}=\exp \left(\mathrm{h}_{p}\right)$. Let $H_{K}=H \cap K$. Then $H=H_{K} \cdot H_{P}$. Let $\mathbf{h}_{\mathrm{C}}^{Z}=\mathbf{g}_{\mathrm{C}}^{Z} \cap \mathbf{h}_{\mathbf{C}}$.

Let $\Phi\left(\mathbf{g}_{\mathbf{C}}, \mathbf{h}_{\mathbf{C}}\right)=\Phi \subset \mathbf{h}_{\mathbf{C}}^{*}$ be the root system for $\mathbf{g}_{\mathbf{C}}$ with respect to $\mathbf{h}_{\mathbf{C}}$. Then $\Phi$ is a root system of type $B_{n}$ :

$$
\Phi=\left\{ \pm\left(e_{i}^{\prime} \pm e_{j}^{\prime}\right), 1 \leq i<j \leq n\right\} \cup\left\{ \pm e_{k}^{\prime}, 1 \leq k \leq n\right\},
$$

where the $e_{k}^{\prime}$ are independent linear forms on $\mathbf{h}_{\mathbf{C}}$. Let $L_{Z}=\left\{H \in \mathbf{h}_{\mathbf{C}} \mid(\alpha, H)\right.$ $\in \mathbf{Z}\}$. Then $\mathbf{h}_{\mathbf{C}}^{Z}=2 \pi i L_{Z}$.

The root system $\Phi$ decomposes into a union $\Phi=\Phi_{L} \cup \Phi_{S}$, where $\Phi_{L}=$ $\left\{ \pm\left(e_{i}^{\prime} \pm e_{j}^{\prime}\right), 1 \leq i<j \leq n\right\}$ are the long roots, and $\Phi_{S}=\left\{ \pm e_{k}^{\prime}, 1 \leq k \leq n\right\}$ are the short roots. A root $\alpha \in \Phi$ is called real if $\left.\alpha\right|_{h}$ is real valued. Denote the set of real roots by $\Phi_{\mathbf{R}}$. For each $\alpha \in \Phi$, denote by $s_{\alpha}$ the Weyl reflection. Let $W \equiv W(\Phi)$ be the Weyl group generated by the $s_{\alpha}, \alpha \in \Phi$. Let $W_{\mathfrak{R}} \equiv W\left(\Phi_{\mathfrak{R}}\right)$ be the subgroup of $W$ generated by $s_{\alpha}, \alpha \in \Phi_{\Re}$.

For each $\alpha \in \Phi_{\mathfrak{R}}$, let $H_{\alpha}$ be the coroot. Choose

$$
X_{\alpha} \in \mathbf{g}_{\alpha}=\{X \in \mathbf{g} \mid(\operatorname{ad} H)(X)=(\alpha, H) X, H \in \mathbf{h}\}
$$

such that $\theta\left(X_{\alpha}\right)=-X_{-\alpha},\left[X_{\alpha}, X_{-\alpha}\right]=H_{\alpha}$ and $\alpha\left(H_{\alpha}\right)=2$. This choice is unique up to a sign. Let $U_{\alpha}=X_{\alpha}-X_{-\alpha}$. Let $m_{\alpha}=\exp \pi\left(U_{\alpha}\right)$. Then the set $\left\{m_{\alpha}, m_{\alpha}^{-1}\right\}$ depends only on $\alpha$ and not on $X_{\alpha}$.

Fix a root $\alpha \in \Phi_{\mathfrak{R}}$. Let $c_{\alpha}=\exp \left(-\frac{i \pi}{4} \operatorname{ad}\left(X_{\alpha}+X_{-\alpha}\right)\right)$ be the Cayley transform associated to $\alpha$. Let $\mathbf{h}_{\alpha}^{\perp}=\{H \in \mathbf{h} \mid(\alpha, H)=0\}$. Let $\mathbf{h}_{\alpha}=\mathfrak{R}\left(X_{\alpha}-X_{-\alpha}\right) \oplus \mathbf{h}_{\alpha}^{\perp}$. Then $\mathbf{h}_{\alpha}$ is called the adjacent Cartan subalgebra to $\mathbf{h}$ with respect to $\alpha$. Note that $\mathbf{h}_{\alpha}=\mathfrak{R}\left(X_{\alpha}-X_{-\alpha}\right) \oplus \mathbf{h}_{k} \oplus \mathbf{h}_{p, \alpha}$, where $\mathbf{h}_{p, \alpha}=\left\{H \in \mathbf{h}_{p} \mid(\alpha, H)=0\right\}$, and that $c_{\alpha}\left(\mathbf{h}_{\mathbf{C}}\right)=\left(\mathbf{h}_{\alpha}\right)_{\mathbf{C}}$.

Let $\Phi\left(\mathbf{h}_{\alpha}\right)=c_{\alpha}(\Phi)$ be the roots of $\mathbf{g}_{\mathbf{C}}$ with respect to $\mathbf{h}_{\alpha, \mathbf{C}}$. Let $\Phi_{\mathfrak{R}}\left(\mathbf{h}_{\alpha}\right)$ be the real roots. Let $\left(\Phi_{\mathfrak{R}}\right)_{\alpha}=\left\{\boldsymbol{\beta} \in \Phi_{\mathfrak{R}} \mid(\boldsymbol{\beta}, \alpha)=0\right\}$. Then $\boldsymbol{\Phi}_{\mathfrak{R}}\left(\mathbf{h}_{\alpha}\right)=c_{\alpha}\left(\left(\Phi_{\mathfrak{R}}\right)_{\alpha}\right)$. Denote by $c_{\alpha}$ the map $c_{\alpha}: W(\Phi) \rightarrow W\left(\Phi\left(\mathbf{h}_{\alpha}\right)\right)$ given by $c_{\alpha} \cdot w=c_{\alpha} w c_{\alpha}^{-1}$.

Define the projection mappings $p_{\mathfrak{R}}: \mathbf{h} \rightarrow \mathbf{h}_{p}$, and $p_{I}: \mathbf{h} \rightarrow \mathbf{h}_{k}$ for any Cartan subalgebra of $\mathbf{g}$. Thus $p_{\mathfrak{R}}: \mathbf{h}_{\alpha} \rightarrow \mathbf{h}_{p, \alpha}$, and $p_{I}: \mathbf{h}_{\alpha} \rightarrow \mathfrak{R}\left(X_{\alpha}-X_{-\alpha}\right) \oplus \mathbf{h}_{k}$. Define the projection mapping $p_{I, \alpha}: \mathbf{h}_{\alpha} \rightarrow \mathfrak{R}\left(X_{\alpha}-X_{-\alpha}\right)$.

Lemma 1.1. Let a be the maximal split Cartan subalgebra of $\mathbf{g}$. Let $Z$ be the center of $G$. Then

(a) $m_{\alpha}^{2}=\varepsilon_{p} \cdot \varepsilon_{q}, \alpha \in \Phi_{\Re, L}$;

(b) $m_{\alpha}=\varepsilon_{q}, \alpha \in \Phi_{\mathfrak{R}, S}$.

Proof. To describe a, we adopt the following notation. Let $I_{p}\left(I_{r}\right)$ be the $p \times p(r \times r)$ identity matrix. Set

$$
J_{p}=\left[\begin{array}{ccc}
0 & I_{p} & 0 \\
I_{p} & 0 & 0 \\
0 & 0 & -I_{r}
\end{array}\right],
$$


$2 p+r-2 n+1$. Let

$$
\begin{aligned}
\operatorname{so}(p, p+r ; \mathfrak{R}) & =\left\{X \in \operatorname{gl}(2 n+1, \mathfrak{R}) \mid X^{t} J_{p}+J_{p} X=0\right\} \\
& =\left\{\left[\begin{array}{ccc}
A & B & C \\
D & -A^{t} & F \\
F^{t} & C^{t} & G
\end{array}\right] \mid \begin{array}{l}
B^{t}+B=D^{t}+D=G^{t}+G=0 \\
A \in \operatorname{gl}(p ; \mathfrak{R}) \\
C, F \in M(p, r ; \mathfrak{R})
\end{array}\right\},
\end{aligned}
$$

where $M(p, r ; \mathfrak{R})$ is any $p \times r$ matrix defined over $\mathfrak{R}$. Then the Cartan decomposition $\mathbf{g}=\mathbf{k} \oplus \mathbf{p}$ is given by

$$
\begin{gathered}
\mathbf{k}=\left\{\left[\begin{array}{ccc}
A & B & C \\
B & -A^{t} & -C \\
-C^{t} & C^{t} & G
\end{array}\right] \mid \begin{array}{l}
A^{t}+A=B^{t}+B=G^{t}+G=0 \\
C \in M(p, r ; \mathfrak{R})
\end{array}\right\}, \\
\mathbf{p}=\left\{\left[\begin{array}{ccc}
A & B & C \\
-B & -A & C \\
C^{t} & C^{t} & 0
\end{array}\right] \mid \begin{array}{l}
A^{t}-A=B^{t}+B=0 \\
C \in M(p, r ; \mathfrak{R})
\end{array}\right\} .
\end{gathered}
$$

The isomorphism $\psi$ between $\mathbf{k}$ and $\operatorname{so}(p) \oplus \operatorname{so}(q)$ is given by

$$
\psi:\left[\begin{array}{ccc}
A & B & C \\
B & -A^{t} & -C \\
-C^{t} & C^{t} & G
\end{array}\right] \mapsto\left[\begin{array}{ccc}
A+B & 0 & 0 \\
0 & A-B & -\sqrt{2 C} \\
0 & \sqrt{2} C^{t} & G
\end{array}\right] .
$$

Let

$$
\mathbf{a}_{p}=\left\{\left[\begin{array}{ccc}
A & 0 & 0 \\
0 & -A & 0 \\
0 & 0 & 0
\end{array}\right] \mid A=\left[\begin{array}{cccc}
h_{1} & & & \\
& h_{2} & & \\
& & \ddots & \\
& & & h_{p}
\end{array}\right], h_{i} \in \mathfrak{R}\right\}
$$

be the maximal abelian subspace of $\mathbf{p}$. Let $\mathbf{m}$ be the centralizer of $\mathbf{a}_{p}$ in $\mathbf{k}$. Let

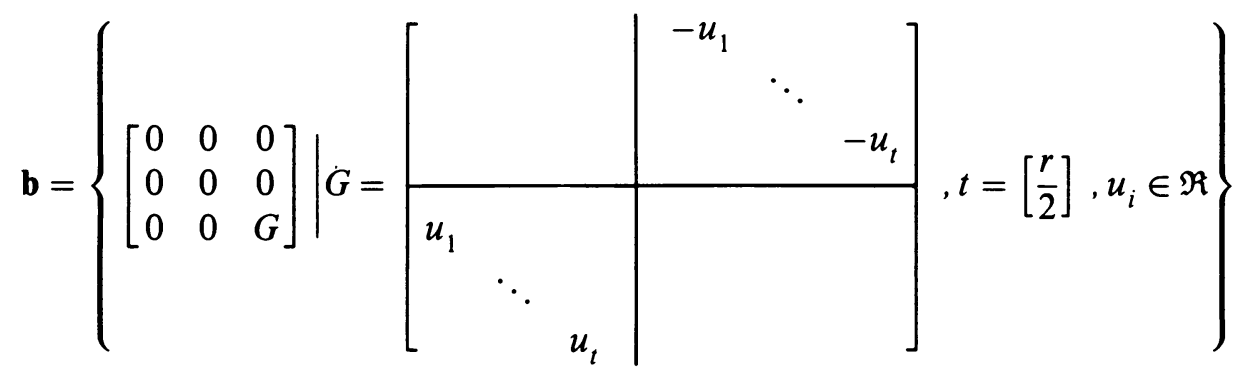

be the maximal abelian subspace of $\mathbf{m}$. Then $\mathbf{a}=\mathbf{a}_{p} \oplus \mathbf{b}$ is the maximal split $\theta$-stable Cartan subalgebra of $\mathbf{g}$. Let $e_{i}^{\prime}$ be the linear form on a defined by

$$
e_{i}^{\prime}(H)=\left\{\begin{array}{ll}
h_{i}, & 1 \leq i \leq p \\
\sqrt{-1} u_{i-p}, & p+1 \leq i \leq n
\end{array}\right\}, \quad H \in \mathbf{a} .
$$

Then the description of $\Phi\left(\mathbf{g}_{\mathbf{C}}, \mathbf{a}_{\mathbf{C}}\right)$ is given by (1.2).

(a) Let $\alpha=e_{i}^{\prime}-e_{j}^{\prime} \in \Phi_{\Re, L}, 1 \leq i, j \leq p$. Let $A$ be the matrix such that

$$
[A]_{k l}=\left\{\begin{array}{ll}
1, & \text { if } k=i, l=j \\
0, & \text { otherwise }
\end{array}\right\} .
$$


Then

$$
\begin{gathered}
X_{\alpha}=\left[\begin{array}{ccc}
A & 0 & 0 \\
0 & -A^{t} & 0 \\
0 & 0 & 0
\end{array}\right], \quad X_{-\alpha}=\left[\begin{array}{ccc}
A^{t} & 0 & 0 \\
0 & -A & 0 \\
0 & 0 & 0
\end{array}\right], \\
U_{\alpha}=\left[\begin{array}{ccc}
A^{\prime} & 0 & 0 \\
0 & A^{\prime} & 0 \\
0 & 0 & 0
\end{array}\right] \in \mathbf{k},
\end{gathered}
$$

where $A^{\prime}=A-A^{t}$. 0 Then under the isomorphism described by (1.3),

$$
m_{\alpha}^{2}=\exp 2 \pi\left[\begin{array}{ccc}
A^{\prime} & 0 & 0 \\
0 & A^{\prime} & 0 \\
0 & 0 & 0
\end{array}\right]=\varepsilon_{p} \cdot \varepsilon_{q} \in Z
$$

(b) Let $\alpha=e_{i}^{\prime}+e_{j}^{\prime} \in \Phi_{\Re, L}, 1 \leq i, j \leq p$. Let $B$ be the matrix such that

$$
[B]_{k l}=\left\{\begin{array}{ll}
1, & k=i, l=j \\
-1, & k=j, l=i \\
0, & \text { otherwise }
\end{array}\right\}
$$

Then

$$
X_{\alpha}=\left[\begin{array}{lll}
0 & B & 0 \\
0 & 0 & 0 \\
0 & 0 & 0
\end{array}\right], \quad X_{-\alpha}=\left[\begin{array}{ccc}
0 & 0 & 0 \\
-B & 0 & 0 \\
0 & 0 & 0
\end{array}\right], \quad U_{\alpha}=\left[\begin{array}{ccc}
0 & B & 0 \\
B & 0 & 0 \\
0 & 0 & 0
\end{array}\right] \in \mathbf{k}
$$

Then under the isomorphism defined by (1.3),

$$
m_{\alpha}^{2}=\exp 2 \pi\left[\begin{array}{ccc}
B & 0 & 0 \\
0 & -B & 0 \\
0 & 0 & 0
\end{array}\right]=\varepsilon_{p} \cdot \varepsilon_{q} \in Z
$$

(c) Let $\alpha=e_{i}^{\prime} \in \Phi_{\Re, S}, \quad 1 \leq i \leq p$. Let $C$ be the matrix such that

$$
[C]_{k l}=\left\{\begin{array}{ll}
1, & k=i, l=2 p+r \\
0, & \text { otherwise }
\end{array}\right\}
$$

Then

$$
\begin{gathered}
X_{\alpha}=\sqrt{2}\left[\begin{array}{lll}
0 & 0 & C \\
0 & 0 & 0 \\
0 & C^{t} & 0
\end{array}\right], \quad X_{-\alpha}=\sqrt{2}\left[\begin{array}{ccc}
0 & 0 & 0 \\
0 & 0 & C \\
C^{t} & 0 & 0
\end{array}\right], \\
U_{\alpha}=\sqrt{2}\left[\begin{array}{ccc}
0 & 0 & C \\
0 & 0 & -C \\
-C^{t} & C^{t} & 0
\end{array}\right] \in \mathbf{k} .
\end{gathered}
$$

Then under the isomorphism defined by (1.3),

$$
m_{\alpha}=\exp 4 \pi\left[\begin{array}{ccc}
0 & 0 & 0 \\
0 & 0 & -C \\
0 & C & 0
\end{array}\right]=\varepsilon_{q} \in Z
$$

Fix $\alpha \in\left(\Phi_{\Re}\right)$. Let $c_{\alpha}$ be the Cayley transform associated to $\alpha$. Let $\beta \in\left(\Phi_{\mathfrak{R}}\right)_{\alpha}$. In order to describe how $m_{\alpha}$ is related to $m_{c_{\kappa} \beta}$ we shall need 
a corresponding result for when $\mathbf{g}$ is the split real Lie algebra of type $B_{2}$. Let $G$ be the universal covering group with Lie $(G)=\mathbf{g}$. Let a be the maximal split Cartan subalgebra. Let $\Delta_{\mathfrak{R}}=\left\{ \pm e_{1}^{\prime} \pm e_{2}^{\prime}, \pm e_{1}^{\prime}, \pm e_{2}^{\prime}\right\}$ be the root system. Fix $\alpha=e_{2}^{\prime} \in \Delta_{\mathfrak{R}}$. Let $c_{\alpha}$ be the corresponding Cayley transform. Let $f_{1}^{\prime}=c_{\alpha}\left(e_{1}^{\prime}\right)$ and $f_{2}^{\prime}=c_{\alpha}\left(e_{2}^{\prime}\right)$.

Lemma 1.2. Let $\alpha=e_{2}^{\prime}$. Then we can choose $m_{f_{1}^{\prime}}, m_{e_{1}^{\prime}}, m_{e_{2}^{\prime}}, m_{e_{1}^{\prime}+e_{2}^{\prime}}$, and $m_{e_{1}^{\prime}-e_{2}^{\prime}}$ such that

(a) $m_{e_{1}^{\prime}}=\left(m_{e_{1}^{\prime}+e_{2}^{\prime}}\right) \cdot\left(m_{e_{1}^{\prime}-e_{2}^{\prime}}\right)^{-1}$;

(b) $m_{f_{1}^{\prime}}=\left(m_{e_{1}^{\prime}+e_{2}^{\prime}}\right) \cdot\left(m_{e_{1}^{\prime}-e_{2}^{\prime}}\right)=\left(m_{e_{1}^{\prime}}\right) \cdot\left(m_{e_{1}^{\prime}-e_{2}^{\prime}}\right)^{2}$.

Let $\alpha=e_{1}^{\prime}-e_{2}^{\prime}$. Then we can choose $m_{f_{1}^{\prime}+f_{2}^{\prime}}$ such that

(c) $m_{f_{1}^{\prime}+f_{2}^{\prime}}=m_{e_{1}^{\prime}+e_{2}^{\prime}}$.

Proof. Apply the coordinates describing the $X_{\alpha}, X_{-\alpha}, U_{\alpha}$ in the proof of Lemma 1.1, to deduce that under the isomorphism given by (1.3),

$$
U_{e_{1}^{\prime}-e_{2}^{\prime}}=\left[\begin{array}{ccccc}
0 & 1 & 0 & 0 & 0 \\
-1 & 0 & 0 & 0 & 0 \\
0 & 0 & 0 & 1 & 0 \\
0 & 0 & -1 & 0 & 0 \\
0 & 0 & 0 & 0 & 0
\end{array}\right] \text {, and } U_{e_{1}^{\prime}+e_{2}^{\prime}}=\left[\begin{array}{ccccc}
0 & 1 & 0 & 0 & 0 \\
-1 & 0 & 0 & 0 & 0 \\
0 & 0 & 0 & -1 & 0 \\
0 & 0 & 1 & 0 & 0 \\
0 & 0 & 0 & 0 & 0
\end{array}\right] \text {. }
$$

Thus

$$
\begin{aligned}
U_{e_{1}^{\prime}+e_{2}^{\prime}}-U_{e_{1}^{\prime}-e_{2}^{\prime}} & =\left[\begin{array}{ccccc}
0 & 0 & 0 & 0 & 0 \\
0 & 0 & 0 & 0 & 0 \\
0 & 0 & 0 & -2 & 0 \\
0 & 0 & 2 & 0 & 0 \\
0 & 0 & 0 & 0 & 0
\end{array}\right], \\
U_{e_{1}^{\prime}+e_{2}^{\prime}}+U_{e_{1}^{\prime}-e_{2}^{\prime}} & =\left[\begin{array}{ccccc}
0 & 2 & 0 & 0 & 0 \\
-2 & 0 & 0 & 0 & 0 \\
0 & 0 & 0 & 0 & 0 \\
0 & 0 & 0 & 0 & 0 \\
0 & 0 & 0 & 0 & 0
\end{array}\right] .
\end{aligned}
$$

Hence $\left(m_{e_{1}^{\prime}+e_{2}^{\prime}}\right) \cdot\left(m_{e_{1}^{\prime}-e_{2}^{\prime}}\right)^{-1}=m_{e_{1}^{\prime}}=m_{e_{2}^{\prime}}$, proving the first statement.

Since $\mathbf{a}_{e_{2}^{\prime}}=\mathfrak{R}\left(U_{e_{2}^{\prime}}\right) \oplus \mathfrak{R} H_{e_{1}^{\prime}}$, we have that

$$
\mathbf{a}_{e_{2}^{\prime}}=\left\{H \in\left[\begin{array}{ccccc}
h_{1} & 0 & 0 & 0 & 0 \\
0 & 0 & 0 & 0 & h_{2} \\
0 & 0 & -h_{1} & 0 & 0 \\
0 & 0 & 0 & 0 & -h_{2} \\
0 & -h_{2} & 0 & h_{2} & 0
\end{array}\right] \mid h_{1}, h_{2} \in \mathfrak{R}\right\}
$$


Then

$$
\begin{gathered}
X_{f_{1}^{\prime}}=\left[\begin{array}{ccccc}
0 & 1 & 0 & 1 & 0 \\
0 & 0 & -1 & 0 & 0 \\
0 & 0 & 0 & 0 & 0 \\
0 & 0 & -1 & 0 & 0 \\
0 & 0 & 0 & 0 & 0
\end{array}\right], X_{-f_{1}^{\prime}}=\left[\begin{array}{cccccc}
0 & 0 & 0 & 0 & 0 \\
1 & 0 & 0 & 0 & 0 \\
0 & -1 & 0 & -1 & 0 \\
1 & 0 & 0 & 0 & 0 \\
0 & 0 & 0 & 0 & 0
\end{array}\right], \\
U_{f_{1}^{\prime}}=\left[\begin{array}{ccccc}
0 & 1 & 0 & 1 & 0 \\
-1 & 0 & -1 & 0 & 0 \\
0 & 1 & 0 & 1 & 0 \\
-1 & 0 & -1 & 0 & 0 \\
0 & 0 & 0 & 0 & 0
\end{array}\right] \in \mathbf{k} .
\end{gathered}
$$

Then under the isomorphism given by (1.3),

$$
U_{f_{1}^{\prime}}=\left[\begin{array}{ccccc}
0 & 2 & 0 & 0 & 0 \\
-2 & 0 & 0 & 0 & 0 \\
0 & 0 & 0 & 0 & 0 \\
0 & 0 & 0 & 0 & 0 \\
0 & 0 & 0 & 0 & 0
\end{array}\right]
$$

Therefore $m_{f_{1}^{\prime}}=\left(m_{e_{1}^{\prime}+e_{2}^{\prime}}\right) \cdot\left(m_{e_{1}^{\prime}-e_{2}^{\prime}}\right)$, proving the second statement.

To prove the last statement, let $\alpha=e_{1}^{\prime}-e_{2}^{\prime}$. Then $\left(\Phi_{\mathfrak{R}}\right)_{\alpha}= \pm\left(e_{1}^{\prime}+e_{2}^{\prime}\right)=\beta$. Since $\alpha \pm \beta$ are not roots, $c_{\alpha}\left(X_{\beta}\right)=X_{\beta}$. Thus we can choose $X_{f_{1}^{\prime}+f_{2}^{\prime}}=X_{e_{1}^{\prime}+e_{2}^{\prime}}$.

We now return to the general setting. Consider the root system $\Phi\left(\mathbf{g}_{\mathbf{C}}, \mathbf{a}_{\mathbf{C}}\right)$ described in the proof of Lemma 1.1. All other $\theta$-stable Cartan subalgebras $\mathbf{h}$ of $\mathbf{g}$ can be obtained from the maximal split Cartan subalgebra a by the Kostant-Sugiura construction [14]. They are in one-to-one correspondence with the conjugacy classes of strongly orthogonal systems of roots. There are two types:

$$
\begin{gathered}
\psi_{1}(j, k)=\left\{e_{1}^{\prime} \pm e_{2}^{\prime}, \ldots, e_{2 j-1}^{\prime} \pm e_{2 j}^{\prime}, e_{2 j+1}^{\prime}-e_{2 j+k+1}^{\prime}, \ldots, e_{2 j+k}^{\prime}-e_{2 j+2 k}^{\prime} \mid\right. \\
0 \leq k, 0 \leq j, 2(j+k) \leq p\} ; \\
\begin{array}{r}
\psi_{2}(j, k)=\left\{e_{1}^{\prime} \pm e_{2}^{\prime}, \ldots, e_{2 j-1}^{\prime} \pm e_{2 j}^{\prime}, e_{2 j+1}^{\prime}-e_{2 j+k+1}^{\prime}, \ldots, e_{2 j+k}^{\prime}\right. \\
\left.-e_{2 j+2 k}^{\prime}, e_{2 j+2 k+1}^{\prime} \mid 0 \leq k, 0 \leq j, 2(j+k)+1 \leq p\right\} .
\end{array}
\end{gathered}
$$

Thus only $\psi_{2}(j, k)$ contains a short root. Denote the conjugacy classes of Cartan subalgebras by $\left[\mathbf{h}_{\psi_{1}}\right]$ and $\left[\mathbf{h}_{\psi_{2}}\right]$ arising from the $\psi_{1}$ and $\psi_{2}$. The root systems $\Phi_{\mathfrak{R}}^{+}$are then of two types:

$$
\begin{aligned}
& \boldsymbol{\Phi}_{\mathfrak{R}}^{+}\left(\left[\mathbf{h}_{\psi_{1}}\right]\right) \simeq A_{1}^{k} \times B_{p-(2 j+2 k)}, \quad 2 j+2 k \leq p, 0 \leq j, k, B_{1} \equiv A_{1} ; \\
& \boldsymbol{\Phi}_{\mathfrak{R}}^{+}\left(\left[\mathbf{h}_{\psi_{2}}\right]\right) \simeq A_{1}^{k} \times B_{p-(2 j+2 k+1)}, \quad 2 j+2 k+1 \leq p, 0 \leq j, k, B_{1} \equiv A_{1} .
\end{aligned}
$$


Lemma 1.3. Let $\mathbf{h}$ be any $\theta$-stable Cartan subalgebra of $\mathbf{g}$. Then

(a) $m_{\alpha}=\left\{\begin{array}{l}\varepsilon_{q}, h \in\left[h_{\psi_{1}}\right] \\ \varepsilon_{p}, h \in\left[h_{\psi_{2}}\right]\end{array}\right\}, \alpha \in \Phi_{\Re, S}$;

(b) $m_{\alpha}^{2}=\varepsilon_{p} \cdot \varepsilon_{q}, \alpha \in \Psi_{\mathfrak{R}, L}$.

Proof. Let $\alpha \in \Phi_{\Re, S}$. If $\mathbf{h} \in\left[\mathbf{h}_{\psi_{2}}\right]$ then there exists a Cayley transform $c_{\beta}$ for $\beta \in \psi_{2}, \beta$ short and $\gamma \in \Phi_{\Re, L}$ such that by Lemma 1.2, $m_{c_{\beta} \alpha}=\left(m_{\alpha}\right) \cdot\left(m_{\gamma}\right)^{2}$. Then by Lemma 1.1, $m_{c_{\beta} \alpha}=\left(\varepsilon_{q}\right) \cdot\left(\varepsilon_{p} \cdot \varepsilon_{q}\right)=\varepsilon_{p}$. If $\mathbf{h} \in\left[\mathbf{h}_{\psi_{1}}\right]$ then $m_{c_{\beta} \alpha}=m_{\alpha}$ for all $\alpha \in \Phi_{\Re, S}$, since $\psi_{1}$ consists entirely of long roots. The second statement holds for all $\mathbf{h}$.

Lemma 1.4. Let $\mathbf{h}$ be any $\theta$-stable Cartan subalgebra of $\mathbf{g}$. Let $\alpha \in \Phi_{\mathfrak{R}}$. Let $c_{\alpha}$ be the Cayley transform corresponding to $\alpha$. Let $\mathbf{h}_{\alpha}$ be the adjacent Cartan subalgebra to $\mathbf{h}$. Let $\chi$ be the fixed character on $Z$.

(a) Fix $\alpha \in \Phi_{\mathfrak{R}, S}$. Let $\beta \in\left(\Phi_{\mathfrak{R}, S}\right)_{\alpha}$. Then $\chi\left(m_{\beta}\right)=-\chi\left(m_{c_{\alpha} \beta}\right)$.

(b) Fix $\alpha \in \Phi_{\mathfrak{R}, L}$. Let $\beta \in\left(\Phi_{\mathfrak{R}, S}\right)_{\alpha}$. Then $\chi\left(m_{\beta}\right)=\chi\left(m_{c_{\alpha} \beta}\right)$.

Proof. Fix $\alpha=e_{n}^{\prime} \in \Phi_{\Re, S}$. Then we are in the situation described by Lemma 1.2. By 1.2(b), $m_{f_{n-1}^{\prime}}=\left(m_{e_{n-1}^{\prime}}\right) \cdot\left(m_{e_{n}^{\prime}-e_{n-1}^{\prime}}\right)^{2}$. Therefore by Lemma 1.1, $\chi\left(m_{f_{n-1}^{\prime}}\right)=-\chi\left(m_{e_{n-1}^{\prime}}\right)$. The second statement follows directly from Lemma $1.2(\mathrm{c})$.

Let $\Phi$ be the root system on $\mathbf{h}_{\mathbf{C}}$. Let $\left\{e_{i} \mid i=1, \ldots, n\right\}$ be the dual basis of $\left\{e_{i}^{\prime} \mid i=1, \ldots, n\right\}$. Let $\Sigma=\left\{ \pm e_{i} \pm e_{j}, 1 \leq i<j \leq n\right\} \cup\left\{ \pm e_{k}, 1 \leq k<n\right\}=$ $\Sigma_{L} \cup \Sigma_{S}$. Then $\Sigma$ is a root system of type $B_{n}$ on the vector space $\mathbf{h}^{*}$. Define a map $\psi$ by

$$
\psi(\alpha)= \begin{cases}H_{\alpha} & \text { for } \alpha \in \Phi_{L}, \\ H_{\alpha} / 2 & \text { for } \alpha \in \Phi_{S}\end{cases}
$$

Then $\psi: \Phi \rightarrow \Sigma$. We will use $\psi$ to identify $\Phi$ and $\Sigma$.

Let $\Phi^{+}$be a choice of positive roots for $\Phi$. Let $\Phi_{\mathfrak{R}}^{+}=\Phi_{\mathfrak{R}} \cap \Phi^{+}$. Let $\Sigma_{\mathfrak{R}}^{+}=\left\{\psi(\alpha) \mid \alpha \in \Phi_{\mathfrak{R}}^{+}\right\}$. Let $\Lambda\left(\Sigma_{\mathfrak{R}}\right)=\sum_{\alpha \in \alpha_{\mathfrak{R}}^{+}} \mathbf{Z} \alpha$. Let $\Lambda^{+}\left(\Sigma_{\mathfrak{R}}\right)=\sum_{\alpha \in \Sigma_{\mathfrak{R}}^{+}} \mathbf{Z}^{+} \alpha$. Then $\Lambda\left(\Sigma_{\mathfrak{R}}\right)$ and $\Lambda^{+}\left(\Sigma_{\mathfrak{R}}\right)$ are lattices $\subset \mathbf{h}_{p}$. Define $\rho_{\mathfrak{R}}^{\Sigma}=\frac{1}{2} \sum_{\alpha \in \Sigma_{\mathfrak{R}}^{+}} \alpha$.

Let $S\left(\Sigma_{\mathfrak{R}}\right)$ be the set of simple roots for $\Sigma_{\mathfrak{R}}^{+}$. Then each root $\beta \in \Sigma_{\mathfrak{R}}^{+}$can be written as $\beta=\sum_{\alpha \in S\left(\Sigma_{\Re)}\right.} k_{\alpha} \alpha, \quad k_{\alpha} \geq 0$. Define the height of a root $\beta \in \Sigma_{\Re}^{+}$ to be $h(\beta)=\Sigma_{\alpha \in S\left(\Sigma_{\Re)}\right.} k_{\alpha}$.

Define a function $\chi_{0}$ on $S\left(\Sigma_{\mathfrak{R}}\right)$ such that $\chi_{0}(\beta)=-1$ for all $\beta \in S\left(\Sigma_{\mathfrak{R}}\right)$. Then $\chi_{0}$ extends to a character on $\Lambda\left(\Sigma_{\mathfrak{R}}\right)$ given by

$$
\chi_{0}(\beta)=(-1)^{h(\beta)}, \quad \beta \in \Lambda\left(\Sigma_{\mathfrak{R}}\right) .
$$

Let $\alpha \in S\left(\Sigma_{\Re}\right)$. Let $\mathbf{h}_{\alpha}^{\prime}$ be the corresponding adjacent Cartan subalgebra. Let $\chi_{0}^{\prime}$ be the character corresponding to $\chi_{0}$ defined on $\Lambda\left(\left(\Sigma_{\mathfrak{R}}\right)_{\alpha}\right) \subset \mathbf{h}_{p, \alpha}$. 
Lemma 1.5. Let $\chi_{0}(\beta)=-1, \beta \in S\left(\Sigma_{\mathfrak{R}}\right)$.

(a) Fix $\alpha \in S\left(\Sigma_{\mathfrak{R}, S}\right)$. Then for $\beta \in\left(\Sigma_{\mathfrak{R}}\right)_{\alpha}$

$$
\chi_{0}(\beta)= \begin{cases}-\chi_{0}^{\prime}\left(c_{\alpha} \beta\right), & \beta \in\left(\Sigma_{\mathfrak{R}, S}\right)_{\alpha}, \\ \chi_{0}^{\prime}\left(c_{\alpha} \beta\right), & \beta \in\left(\Sigma_{\mathfrak{R}, L}\right)_{\alpha} .\end{cases}
$$

(b) Fix $\alpha \in S\left(\Sigma_{\mathfrak{R}, L}\right)$. Then

$$
\chi_{0}(\beta)=\chi_{0}^{\prime}\left(c_{\alpha} \beta\right), \quad \beta \in\left(\Sigma_{\mathfrak{R}}\right)_{\alpha} .
$$

Proof. Let $\alpha \in S\left(\Sigma_{\Re, S}\right)$. Let $h^{\prime}$ be the corresponding height function on $\mathbf{h}_{\alpha}$. Then for $\beta \in\left(\Sigma_{\mathfrak{R}, S}\right)_{\alpha}, h^{\prime}(\beta)=h\left(c_{\alpha}^{-1}(\beta)\right)-1$, and for $\beta \in\left(\Sigma_{\mathfrak{R}, L}\right)_{\alpha}, h^{\prime}(\beta)=$ $h\left(c_{\alpha}^{-1}(\beta)\right) \bmod 2$. Let $\alpha \in S\left(\Sigma_{\mathfrak{R}, L}\right)$. Then for $\beta \in\left(\Sigma_{\mathfrak{R}}\right)_{\alpha}, h^{\prime}(\beta)=h\left(c_{\alpha}^{-1}(\beta)\right)$ $\bmod 2$.

Let $\chi$ be the character on $Z$. Define a function $\chi_{1}$ on $\Sigma_{\Re}$ by

$$
\chi_{1} \beta= \begin{cases}-\chi_{0}(\beta) \cdot \chi\left(m_{\psi^{-1}(\beta)}\right), & \beta \in \Sigma_{\mathfrak{R}, S}, \\ \chi_{0}(\beta), & \beta \in \Sigma_{\mathfrak{R}, L} .\end{cases}
$$

Lemma 1.6. $\chi_{1}$ extends to a well-defined character on $\Lambda\left(\Sigma_{\mathfrak{R}}\right)$.

Proof. Let $\alpha, \beta \in \Sigma_{\mathfrak{R}, S}$ such that $\alpha+\beta \in \Sigma_{\mathfrak{R}, L}$. Then

$$
\chi_{1}(\alpha) \chi_{1}(\beta)=\chi_{0}(\alpha+\beta) \chi\left(m_{\psi^{-1}(\alpha)}\right) \chi\left(m_{\psi^{-1}(\beta)}\right)=\chi_{1}(\alpha+\beta)
$$

since $m_{\psi^{-1}(\alpha)} \cdot m_{\psi^{-1}(\beta)}=1$. Let $\alpha \in \Sigma_{\mathfrak{R}, S}, \beta \in \Sigma_{\mathfrak{R}, L}$ such that $\alpha+\beta \in \Sigma_{\mathfrak{R}, S}$. Then

$$
\begin{aligned}
\chi_{1}(\alpha) \chi_{1}(\beta) & =(-1) \chi_{0}(\alpha+\beta) \chi\left(m_{\psi^{-1}(\alpha)}\right) \\
& =(-1) \chi_{0}(\alpha+\beta) \chi\left(m_{\psi^{-1}(\alpha+\beta)}\right)=\chi_{1}(\alpha+\beta) .
\end{aligned}
$$

Finally, let $\alpha, \beta \in \Sigma_{\mathfrak{R}, L}$ such that $\alpha+\beta \in \Sigma_{\mathfrak{R}, L}$. Then

$$
\chi_{1}(\alpha) \chi_{1}(\beta)=\chi_{0}(\alpha+\beta)=\chi_{1}(\alpha+\beta) \text {. }
$$

Lemma 1.7. Let $\beta \in\left(\Sigma_{\mathfrak{R}}\right)_{\alpha}$. Then $\chi_{1}^{\mathrm{h}}(\beta)=\chi_{1}^{\mathrm{h}_{1}}\left(c_{\alpha} \beta\right)$.

Proof. By Lemma 1.4, Lemma 1.5, and the definition of $\chi_{1}(\beta)$ given in (1.6), the statement of Lemma 1.7 holds.

Lemma 1.8. Let $\alpha \in S\left(\Sigma_{\Re}\right)$. Then $\chi_{1}(\alpha)=\chi\left(\exp \left(2 \pi P_{I, \alpha}\left(c_{\alpha} \alpha\right)\right)\right)$.

Proof. Let $\alpha \in S\left(\Sigma_{\Re, S}\right)$. Then

$$
\chi_{1}(\alpha)=\chi\left(m_{\psi^{-1}(\alpha)}\right)=\chi\left(\exp \left(2 \pi\left(X_{\alpha}-X_{-\alpha}\right) / 2\right)\right)=\chi\left(\exp \left(2 \pi P_{I, \alpha}\left(c_{\alpha} \alpha\right)\right)\right) .
$$

Let $\alpha \in S\left(\Sigma_{\Re, L}\right)$. Then $\chi_{1}(\alpha)=-1$, and $\chi\left(\exp \left(2 \pi\left(X_{\alpha}-X_{-\alpha}\right)\right)\right)=\chi\left(m_{\psi^{-1}(\alpha)}^{2}\right)$ $=-1$ by the definition of $\chi$.

Define the function $\tilde{\chi}$ on $\Sigma_{\Re}^{+}$by $\tilde{\chi}(\beta)=\chi_{0}(\beta) \chi_{1}(\beta)$. Then

$$
\tilde{\chi}(\beta)= \begin{cases}-\chi\left(m_{\psi^{-1}(\beta)}\right), & \beta \in \Sigma_{\mathfrak{R}, S}^{+}, \\ 1, & \beta \in \Sigma_{\mathfrak{R}, L}^{+} .\end{cases}
$$

Since $\chi_{0}$ and $\chi_{1}$ extend to well-defined characters on $\Lambda\left(\Sigma_{\mathfrak{R}}\right), \tilde{\chi}$ extends to a well-defined character on $\Lambda\left(\Sigma_{\mathfrak{R}}\right)$. 
Lemma 1.9. Let $\xi \in \mathbf{h}_{\mathbf{C}}^{Z}, \xi=2 \pi\left(\xi_{0}+i \xi_{1}\right)$, such that $P_{\mathfrak{R}}\left(c_{\alpha} \xi_{1}\right) \in \Lambda\left(\Sigma_{\mathfrak{R}}\left(\mathbf{h}_{\alpha}\right)\right)$. Then $\chi_{1}\left(\xi_{1}\right)=\chi\left(\exp \left(2 \pi P_{I, \alpha}\left(c_{\alpha} \xi_{1}\right)\right)\right) \chi_{1}^{\mathbf{h}_{i}}\left(P_{\mathfrak{R}}\left(c_{\alpha} \xi_{1}\right)\right)$.

Proof. Write $c_{\alpha} \xi_{1}=P_{I, \alpha}\left(c_{\alpha} \xi_{1}\right)+P_{\mathfrak{R}}\left(c_{\alpha} \xi_{1}\right)$. If $P_{\mathfrak{R}}\left(c_{\alpha} \xi_{1}\right) \in \Lambda\left(\Sigma_{\mathfrak{R}}\left(\mathbf{h}_{\alpha}\right)\right)$, then $\xi_{1} \in\left(\mathbf{Z} \alpha \oplus \sum_{\beta \in\left(\Sigma_{\mathfrak{R}}^{+}\right)_{\mathfrak{R}}} \mathbf{Z} \beta\right)$. Since $\alpha \in S\left(\Sigma_{\mathfrak{R}}\right)$ and $\beta \in\left(\Sigma_{\mathfrak{R}}^{+}\right)_{\alpha}$, we can apply Lemma 1.7 and Lemma 1.8 with $\xi_{1}=(m \alpha+\gamma)$, where $\gamma \in \sum_{\beta \in\left(\Sigma_{\mathfrak{R}}^{+}\right)_{\alpha}} \mathbf{Z} \beta$, $m \in \mathbf{Z}, P_{\mathfrak{R}}\left(c_{\alpha} \xi_{1}\right)=\gamma$, and $P_{I, \alpha}\left(c_{\alpha} \xi_{1}\right)=m c_{\alpha} \alpha$.Then,

$$
\begin{aligned}
\chi_{1}(m \alpha+\gamma) & =\chi_{1}(m \alpha) \chi_{1}(\gamma)=\chi\left(\exp \left(2 \pi m P_{I, \alpha}\left(c_{\alpha} \alpha\right)\right)\right) \chi_{1}^{\mathbf{h}_{i \prime}}(\gamma) \\
& =\chi\left(\exp \left(2 \pi P_{I, \alpha}\left(c_{\alpha} \xi_{1}\right)\right)\right) \chi_{1}^{\mathbf{h}_{i \prime}}\left(P_{\mathfrak{R}}\left(c_{\alpha} \xi_{1}\right)\right),
\end{aligned}
$$

proving the lemma.

In this section, we shall state Theorem 2.1, due to D. Peterson. We then describe the Plancherel product formula. Proposition 2.2 enables us to expand the Plancherel product formula as a power series expansion. Then we use results from $\S 1$ and the D. Peterson theorem to prove Proposition 2.4, which will be the main technical result necessary to prove the Poisson-Plancherel formula.

We shall now state a theorem due to D. Peterson.

Let $\Phi$ be the root system on a vector space $V$. Let $\Phi^{+}$be a choice of positive roots. Let $S(\Phi)$ be the set of simple roots.

Define the function on $V$

$$
P(H)=\prod_{\beta \in \Phi^{+}} \frac{1-e^{-(\beta, H)}}{1+e^{-(\beta, H)}}, \quad H \in V .
$$

If $H$ is such that $(\beta, H)>0$, then there is the equality of convergent series

$$
\frac{1}{1+e^{-(\beta, H)}}=\sum_{n \geq 0}(-1)^{n} e^{-n(\beta, H)}
$$

Let $C^{+}=\left\{H \in V \mid(\beta, H)>0, \beta \in \Phi^{+}\right\}$. Let $\Lambda^{+}(\Phi)=\sum_{\alpha \in \Phi^{+}} \mathbf{Z}^{+} \alpha$. Define the constants $d\left(\mu ; \Phi^{+}\right)$by the expansion

$$
P(H)=\sum_{\mu \in \Lambda^{+}(\Phi)} d\left(\mu ; \Phi^{+}\right) e^{-(\mu, H)}, \quad H \in C^{+} .
$$

Extend the constants $d\left(\mu ; \Phi^{+}\right)$to all of $V^{*}$ by setting $d\left(\mu ; \Phi^{+}\right)=0$ if $\mu \notin$ $\sum_{\alpha \in \Phi^{+}} \mathbf{Z}^{+} \alpha$.

Fix $\alpha \in S(\Phi)$. Let $\Phi_{\alpha}=\{\beta \in \Phi \mid(\beta, \alpha)=0\}$. Let $\Phi_{\alpha}^{+}=\Phi^{+} \cap \Phi_{\alpha}$. Let $V_{\alpha}=\{v \in V \mid(v, \alpha)=0\}$. Let $u_{\alpha}$ denote the restriction of $\mu \in V^{*}$ to $V_{\alpha}$. Let $\chi_{0}^{\Phi^{+}}$be the character of $\Lambda(\Phi)$ such that $\chi_{0}^{\Phi^{+}}(\beta)=-1$ for all $\beta \in S(\Phi)$. 
Theorem 2.1 [Peterson]. Let $\Phi$ be a root system on a vector space $V$ and $\Phi^{+}$ be a choice of positive roots. Fix a simple root $\alpha \in S(\Phi)$ and let $s_{\alpha} \in W(\Phi)$ be the Weyl reflection associated to $\alpha$. Then for $\mu \in \Lambda(\Phi)$,

$$
\chi_{0}^{\Phi^{+}}(\mu)\left(d\left(\mu ; \Phi^{+}\right)+d\left(s_{\alpha} \mu ; \Phi^{+}\right)\right)=2 \chi_{0}^{\Phi_{\text {"i }}^{+}}\left(\mu_{\alpha}\right) d\left(\mu_{\alpha} ; \Phi_{\alpha}^{+}\right)
$$

Proof. See [12].

We shall now describe the Plancherel product formula.

Let $H=H_{K} H_{p}$ by the Cartan subgroup of $G$ associated to $\mathbf{h}$. Define the subset of irreducible representations of $H$

$$
\hat{H}(\chi)=\{\tau \in \hat{H} \mid \tau(z)=\chi(z) \cdot \text { Id }, z \in Z\} .
$$

For $\tau \in \hat{H}$, define $b \in \hat{H}_{K}$ and $\nu \in \mathbf{h}_{p}^{*}$ by the relations

$$
\left.\tau\right|_{H_{K}}=b, \quad \tau(\exp X)=e^{i(\nu, X)}, \quad X \in \mathbf{h}_{p} .
$$

Let $\Phi_{\mathfrak{R}}^{+}$be a choice of positive real roots. For each $\alpha \in \Phi_{\mathfrak{R}}^{+}$, and $\tau \in \hat{H}(\chi)$, let $P_{\alpha}(\tau) \equiv P_{\alpha}(b, \nu)$ be the Plancherel function defined by Harish-Chandra [8] and given by the matrix

$$
P_{\alpha}(b, \nu)=\left[\frac{\operatorname{sh}\left(\pi \nu, H_{\alpha}\right) \cdot \mathrm{Id}}{\operatorname{ch}\left(\pi \nu, H_{\alpha}\right) \cdot \mathrm{Id}+(-1)^{\left(\rho_{\mathscr{P}}^{+}, H_{\alpha}\right)} \cdot \frac{\left(b\left(m_{\alpha}\right)+b\left(m_{\alpha}\right)^{-1}\right)}{2}}\right] .
$$

The group $H$ is nonabelian, but results of Duflo [5] show that $b\left(m_{\alpha}\right)+$ $b\left(m_{\alpha}\right)^{-1}$ is a scalar matrix. Thus the Plancherel product formula for the Plancherel function can be written as

$$
P(b, \nu)=\frac{1}{(\operatorname{dim} b)^{\# \Phi_{\mathfrak{R}}^{+}}} \prod_{\alpha \in \Phi_{\mathfrak{\Re}}^{+}} \operatorname{tr} P_{\alpha}(b, \nu) .
$$

Proposition 2.2. Let $g=\operatorname{so}(p, q ; \mathfrak{R}), p+q=2 n+1$ and $p$ or $q \neq 0,1,2$. Let $\mathbf{h}=\mathbf{h}_{k} \oplus \mathbf{h}_{p}$ be a $\theta$-stable Cartan subalgebra of $\mathbf{g}$. Let $\psi: \Phi_{\mathfrak{R}}^{+} \rightarrow \Sigma_{\mathfrak{R}}^{+}$be the map given by (1.5). Let $\tilde{\chi}$ be the character on $\Lambda\left(\Sigma_{\mathfrak{R}}\right)$ defined by (1.7). Then for $b \in \hat{H}_{K}(\chi)=\hat{H}(\chi) \cap \hat{H}_{K}$

$$
P(b, \nu)=\prod_{\beta \in \Sigma_{\Re}^{+}} \frac{1-\tilde{\chi}(\beta) e^{-(2 \pi \nu, \beta)}}{1+\tilde{\chi}(\beta) e^{-(2 \pi \nu, \beta)}}
$$

Proof. By (2.3)

$$
P(b, \nu)=\frac{1}{(\operatorname{dim} b)^{\| \Phi_{\Re}^{+}}} \prod_{\alpha \in \Phi_{\mathscr{R}}^{+}} \operatorname{tr}\left[\frac{\operatorname{sh}\left(\pi \nu, H_{\alpha}\right) \cdot \mathrm{Id}}{\operatorname{ch}\left(\pi \nu, H_{\alpha}\right) \cdot \mathrm{Id}+(-1)^{\left(\rho_{\mathscr{N}}^{+}, H_{\alpha}\right)} \cdot\left(b(m(\alpha))+b(m(\alpha))^{-1}\right) / 2}\right]
$$

When $\alpha \in \Phi_{\mathfrak{R}, L}^{+}, \chi\left(m(\alpha)^{2}\right)=-1$. Therefore $b(m(\alpha))+b(m(\alpha))^{-1}=0$, since $b(m(\alpha))$ has $\pm i$ eigenvalues for $\alpha \in \Phi_{\mathfrak{R}, L}^{+}$. When $\alpha \in \Phi_{\mathfrak{R}, S}^{+}$, we have 
that $(-1)^{\left(\rho_{\mathfrak{R}}^{\Phi^{+}}, H_{n}\right)}=-1$ and $b(m(\alpha))+b(m(\alpha))^{-1} / 2=\chi(m(\alpha)) \cdot$ Id . Therefore $P(b, \nu)$ becomes

$$
P(b, \nu)=\prod_{\alpha \in \Phi_{\mathfrak{R}, L}} \frac{\operatorname{sh}\left(\pi \nu, H_{\alpha}\right)}{\operatorname{ch}\left(\pi \nu, H_{\alpha}\right)} \cdot \prod_{\alpha \in \Phi_{\mathscr{R}}^{+}, S} \frac{\operatorname{sh}\left(\pi \nu, H_{\alpha}\right)}{\operatorname{ch}\left(\pi \nu, H_{\alpha}\right)-\chi(m(\alpha))} .
$$

Let $\psi: \Phi_{\mathfrak{R}}^{+} \rightarrow \Sigma_{\mathfrak{R}}^{+}$be the map given by (1.5). Since $\psi(\alpha)=H_{\alpha}$ for $\alpha \in$ $\Phi_{\mathfrak{R}, L}^{+}$, and $\psi(\alpha)=H_{\alpha} / 2$ for $\alpha \in \Phi_{\mathfrak{R}, S}^{+}$, we have that

$$
P(b, \nu)=\prod_{\alpha \in \Sigma_{\mathscr{N}, L}^{+}} \frac{1-e^{-(2 \pi \nu, \alpha)}}{1+e^{-(2 \pi \nu, \alpha)}} \cdot \prod_{\beta \in \Sigma_{\mathscr{R}, S}^{+}} \frac{1+\chi\left(m_{\psi^{-1}(\beta)}\right) e^{-(2 \pi \nu, \beta)}}{1-\chi\left(m_{\psi^{-1}(\beta)}\right) e^{-(2 \pi \nu, \beta)}}
$$

Finally, let $\tilde{\chi}(\beta)$ be the character defined by (1.7). Then

$$
P(b, \nu)=\prod_{\beta \in \Sigma_{\Re}^{+}} \frac{1-\tilde{\chi}(\beta) e^{-(2 \pi \nu, \beta)}}{1+\tilde{\chi}(\beta) e^{-(2 \pi \nu, \beta)}}
$$

Define the constants $d\left(\xi ; \Sigma_{\mathfrak{R}}^{+}\right)$in the identical manner as in (2.1), replacing $\Phi_{\mathfrak{R}}^{+}$by $\Sigma_{\mathfrak{R}}^{+}$, where $V=\mathbf{h}_{p}^{*}$. Let $\Lambda^{+}\left(\Sigma_{\mathfrak{R}}\right)=\sum_{\alpha \in \Sigma_{\mathfrak{R}}^{+}} \mathbf{Z}^{+} \alpha$. Then a formal power series expansion of (2.4) gives

$$
P(b, \nu)=\sum_{\xi \in \Lambda^{+}\left(\Sigma_{\mathfrak{R}}\right)} \tilde{\chi}(\xi) d\left(\xi ; \Sigma_{\mathfrak{R}}^{+}\right) e^{-(2 \pi \nu, \xi)} .
$$

Let $\mathbf{h}=\mathbf{h}_{k} \oplus \mathbf{h}_{p}$ be the $\theta$-stable Cartan decomposition of $\mathbf{h}$. Let $\xi \in L_{Z}$, $\xi=i \xi_{0}+\xi_{1}$, where $\xi_{0} \in P_{I}\left(\mathbf{h}_{\mathbf{C}}\right)$ and $\xi_{1} \in P_{\mathfrak{R}}\left(\mathbf{h}_{\mathbf{C}}\right)$.

Define $\mathbf{h}^{*}(\chi)=\left\{\lambda \in \mathbf{h}^{*} \mid e^{i(\lambda, \delta)}=\chi(\exp \delta)\right.$ for $\left.\delta \in \mathbf{h}_{Z}\right\}$. Let $\mathbf{h}_{k}^{*}(\chi)=$ $\mathbf{h}^{*}(\chi) \cap \mathbf{h}_{k}^{*}$. Let $\mu \in \mathbf{h}_{k}^{*}(\chi)$. Define the function on $L_{Z}$

$$
m^{\mu}\left(\chi: \xi ; \Phi_{\mathfrak{R}}^{+}\right)=e^{i\left(2 \pi \xi_{0}, \mu\right)} d\left(\xi_{1} ; \Sigma_{\mathfrak{R}}^{+}\right) \tilde{\chi}\left(\xi_{1}\right) .
$$

Lemma 2.3. If $\xi \in L_{Z}, \xi=i \xi_{0}+\xi_{1}$, then

(a) $m^{\mu}\left(\chi: \xi ; \Phi_{\mathfrak{R}}^{+}\right)=0$ if $\xi_{1} \notin \Lambda\left(\Sigma_{\mathfrak{R}}\right)$.

(b) If $\xi_{1} \in \Lambda\left(\Sigma_{\mathfrak{R}}\right)$, then $2 \pi \xi_{0} \in \mathbf{h}^{Z}$ and

$$
m^{\mu}\left(\chi: \xi ; \Phi_{\mathfrak{R}}^{+}\right)=\chi\left(\exp \left(2 \pi \xi_{0}\right)\right) \tilde{\chi}\left(\xi_{1}\right) d\left(\xi_{1} ; \Sigma_{\mathfrak{R}}^{+}\right)
$$

Proof. The first statement follows directly from the definitions of the constants $d\left(\xi_{1} ; \Sigma_{\mathfrak{R}}^{+}\right)$which vanish when $\xi_{1} \notin \Lambda\left(\Sigma_{\mathfrak{R}}\right)$.

In the second statement, since $\xi \in L_{Z}$ and $\xi_{1} \in \Lambda\left(\Sigma_{\Re}\right)$, then $2 \pi i \xi_{1} \in \mathbf{h}_{\mathbf{C}}^{Z}$. Hence $2 \pi \xi_{0} \in \mathbf{h}_{\mathbf{C}}^{\mathbf{Z}}$, and $e^{i\left(2 \pi \xi_{0}, \mu\right)}=\chi\left(\exp \left(2 \pi \xi_{0}\right)\right)$.

In particular, if $\xi \in L_{Z}, m^{\mu}\left(\chi: \xi ; \Phi_{\Re}^{+}\right)$is independent of the choice of $\mu \in$ $\mathbf{h}^{*}(\chi)$. Denote this value by $m^{\chi}\left(\xi ; \Phi_{\mathfrak{R}}^{+}\right)$, for $\xi \in L_{Z}$. 
Proposition 2.4 [notation of $\S 1$ ]. Let $\xi \in L_{Z}$ such that $\xi=i \xi_{0}+\xi_{1}$. Then $m^{\chi}\left(\xi: \Phi_{\mathfrak{R}}^{+}\right)+m^{\chi}\left(s_{\alpha} \xi ; \Phi_{\mathfrak{R}}^{+}\right)=m^{\chi}\left(c_{\alpha} \xi ; \boldsymbol{\Phi}_{\mathfrak{R}}^{+}\left(\mathbf{h}_{\alpha}\right)\right)+m^{\chi}\left(c_{\alpha} s_{\alpha} \xi ; \boldsymbol{\Phi}_{\mathfrak{R}}^{+}\left(\mathbf{h}_{\alpha}\right)\right)$.

Proof. By the isomorphism between $\psi$ and $\Sigma$, identify $\psi(\alpha) \equiv \alpha \in \Sigma_{\mathfrak{R}}$. Fix $\alpha \in S\left(\Sigma_{\mathfrak{R}}\right)$. By the preceding lemma, the left-hand side (LHS) of the proposition becomes

$$
\text { LHS }=\chi\left(\exp \left(2 \pi \xi_{0}\right)\right) \tilde{\chi}\left(\xi_{1}\right) d\left(\xi_{1} ; \Sigma_{\mathfrak{R}}^{+}\right)+\chi\left(\exp \left(2 \pi s_{\alpha} \xi_{0}\right)\right) \tilde{\chi}\left(s_{\alpha} \xi_{1}\right) d\left(s_{\alpha} \xi_{1} ; \Sigma_{\mathfrak{R}}^{+}\right) .
$$

Since $\alpha$ is a real root, $s_{\alpha}$ leaves $\mathbf{h}_{k}$ fixed and $s_{\alpha}$ leaves the decomposition of $\Sigma_{\mathfrak{R}}^{+}=\Sigma_{\mathfrak{R}, L}^{+} \cup \Sigma_{\mathfrak{R}, S}^{+}$stable. Using $\tilde{\chi}(\beta)=\chi_{0}(\beta) \chi_{1}(\beta)$, the LHS becomes

$$
\text { LHS }=\chi\left(\exp 2 \pi \xi_{0}\right) \chi_{1}\left(\xi_{1}\right) \chi_{0}\left(\xi_{1}\right)\left(d\left(\xi_{1} ; \Sigma_{\mathfrak{R}}^{+}\right)+d\left(s_{\alpha} \xi_{1} ; \Sigma_{\mathfrak{R}}^{+}\right)\right) \text {. }
$$

Using the Peterson Theorem 2.1, the LHS becomes

$$
\text { LHS }=2 \chi\left(\exp 2 \pi \xi_{0}\right) \chi_{1}\left(\xi_{1}\right) \chi_{0}^{\Sigma_{\mathfrak{R}}^{+}\left(\mathbf{h}_{\mathfrak{r}}\right)}\left(P_{\mathfrak{R}}\left(c_{\alpha} \xi_{1}\right)\right) d\left(P_{\mathfrak{R}}\left(c_{\alpha} \xi_{1} ; \Sigma_{\mathfrak{R}}^{+}\left(\mathbf{h}_{\alpha}\right)\right)\right) \text {. }
$$

Now consider the right-hand side (RHS) of the proposition. The element $c_{\alpha} \xi_{1}=P_{I, \alpha}\left(c_{\alpha} \xi_{1}\right)+P_{\mathfrak{R}}\left(c_{\alpha} \xi_{1}\right)$. Also $P_{I, \alpha}\left(c_{\alpha}\left(s_{\alpha} \xi_{1}\right)\right)=P_{I, \alpha}\left(-c_{\alpha} \xi_{1}\right)$ and $P_{\mathfrak{R}}\left(c_{\alpha} s_{\alpha} \xi_{1}\right)$ $=P_{\mathfrak{R}}\left(c_{\alpha} \xi_{1}\right)$. Therefore the RHS becomes

$$
\begin{aligned}
\text { RHS }= & 2 \chi\left(\exp 2 \pi \xi_{0}\right) \chi\left(\exp \left(2 \pi P_{I, \alpha}\left(c_{\alpha} \xi_{1}\right)\right)\right) \\
& \times \tilde{\chi}^{\mathbf{h}_{\boldsymbol{h}}}\left(P_{\mathfrak{R}}\left(c_{\alpha} \xi_{1}\right)\right) d\left(P_{\mathfrak{R}}\left(c_{\alpha} \xi_{1}\right) ; \Sigma_{\mathfrak{R}}^{+}\left(\mathbf{h}_{\alpha}\right)\right) .
\end{aligned}
$$

Since $\tilde{X}^{\mathbf{h}_{\text {h }}}$ is a character on the lattice $\Lambda\left(\Sigma_{\mathfrak{R}}\left(\mathbf{h}_{\alpha}\right)\right), \tilde{\chi}^{\mathbf{h}_{\alpha}}(\boldsymbol{\beta})=\chi_{0}^{\mathbf{h}_{\alpha}}(\beta) \chi_{1}^{\mathbf{h}_{\alpha}}(\boldsymbol{\beta})$, $\beta \in \Lambda\left(\Sigma_{\mathfrak{R}}\left(\mathbf{h}_{\alpha}\right)\right)$. Thus

$$
\begin{aligned}
\text { RHS }= & 2\left(\exp 2 \pi \xi_{0}\right) \chi\left(\exp \left(2 \pi P_{I, \alpha}\left(c_{\alpha} \xi_{1}\right)\right)\right) \\
& \times \chi_{0}^{\mathbf{h}_{\alpha}}\left(P_{\mathfrak{R}}\left(c_{\alpha} \xi_{1}\right)\right) \chi_{1}^{\mathbf{h}_{\alpha}}\left(P_{\mathfrak{R}}\left(c_{\alpha} \xi_{1}\right)\right) d\left(P_{\mathfrak{R}} c_{\alpha}\left(\xi_{1}\right) ; \Sigma_{\mathfrak{R}}^{+}\left(\mathbf{h}_{\alpha}\right)\right) .
\end{aligned}
$$

Recall that the constants $d\left(P_{\mathfrak{R}}\left(c_{\alpha} \xi_{1}\right) ; \Sigma_{\mathfrak{R}}^{+}\left(\mathbf{h}_{\alpha}\right)\right)=0$, when $P_{\mathfrak{R}}\left(c_{\alpha} \xi_{1}\right) \notin$ $\Lambda\left(\Sigma_{\mathfrak{R}}\left(\mathbf{h}_{\alpha}\right)\right)$. Therefore the proof of Proposition 2.4 follows directly from Lemma 1.9. Q.E.D.

In this section we will describe the setting for the Poisson-Plancherel formula and then use earlier results to prove the formula in Theorems 3.4 and 3.5.

Let $\mathbf{h}$ be a Cartan subalgebra of $\mathbf{g}$. Let $\Pi_{\mathbf{h}}(H)=\prod_{\alpha \in \Phi^{+}}(\alpha, H)$. Let $\mathbf{h}_{r}=$ $\left\{H \in \mathbf{h} \mid \Pi_{\mathbf{h}}(H) \neq 0\right\}$ be the regular set of elements of $\mathbf{h}$.

Choose Lebesgue measures $d X$ on $\mathrm{g}$ and $d H$ on $\mathbf{h}$ such that the Haar measure $d \dot{g}$ on $G / H$ is normalized by

$$
\int_{G \cdot \mathbf{h}_{r}} \phi(X) d X=\int_{\mathbf{h}_{r}}|\Pi(H)|^{2} \int_{G / H} \phi(g \cdot H) d \dot{g} d H,
$$

where $g \cdot H$ denotes $(\operatorname{Ad} g)(H)$ for $g \in G, H \in \mathbf{h}_{r}$.

Normalize the Lebesgue measure $d X$ as follows. Define the Fourier transforms on $g$ by

$$
\hat{\phi}_{g}(f)=\int_{\mathbf{g}} e^{i(f, X)} \phi(X) d X
$$


where the Lebesgue measure $d X$ is normalized such that $\hat{\phi}_{\mathbf{g}}^{2}(X)=\phi(-X)$ for rapidly decreasing functions $\phi$.

Let

$$
\mathbf{h}_{r}^{*}=\left\{\lambda \in \mathbf{h}^{*} \mid \prod_{\alpha \in \Phi^{+}}(\alpha, \lambda) \neq 0\right\}
$$

be the regular elements in $\mathbf{h}^{*}$. For $\lambda \in \mathbf{h}_{r}^{*}$, let $G \cdot \lambda=\Omega$ be the co-adjoint orbit through $\lambda$. Define the canonical measure $\mu_{\Omega}$ on $\Omega$ as follows. Let $\sigma_{\lambda}(X, Y)=(\lambda,[X, Y])$ be the skew form on the tangent space $T_{\lambda} \Omega$. Let $\left|\operatorname{det} \sigma_{\lambda}\left(u_{i}, u_{j}\right)\right|^{1 / 2}$ be the volume of the parallelepiped spanned by the basis elements $\left\{u_{i}\right\}$ of $T_{\lambda} \Omega$. This determines a smooth $G$-invariant measure on $\Omega$.

We normalize the measure $d \dot{g}$ on $G / H$ such that

$$
(k \phi)(\lambda)=\int_{\Omega} \phi(f) d \mu_{\Omega}(f)=(2 \pi)^{-d}\left|\Pi_{\mathbf{h}}(\lambda)\right| \int_{G / H} \phi(g \cdot \lambda) d \dot{g},
$$

where $\operatorname{dim} G / H=2 d$.

Let $\mathbf{t}$ be a maximal abelian subalgebra of $\mathbf{g}$ contained in $\mathbf{k}$. Then $\mathbf{t}$ is a Cartan subalgebra of $\mathbf{g}$. Let $T$ be the Cartan subgroup with Lie algebra $\mathbf{t}$. Let $W_{K}$ be the Weyl group associated to the roots of $\mathbf{t}_{\mathbf{C}}$ in $\mathbf{k}_{\mathbf{C}}$. Let $\Phi \in \mathscr{S}(g)$. Define

$$
\left(M_{\mathbf{t}} \phi\right)(H)=\Pi_{\mathbf{t}}(H) \int_{G / T} \phi(g \cdot H) d \dot{g}, \quad h \in \mathbf{t}_{r} .
$$

By the results of Harish-Chandra [6], the Fourier transform of the distribution $\phi \rightarrow\left(M_{t} \phi\right)(H)$ is given by

$$
\left(M_{\mathbf{t}} \phi\right)(H)=\int_{\mathbf{g}^{*}} \theta(H ; \lambda) \hat{\phi}_{\mathbf{g}}(\lambda) d \lambda, \quad H \in \mathbf{t}_{r},
$$

where $\theta(H ; \lambda)$ is a $G$-invariant, locally $L^{1}$, function on $\mathbf{g}^{*}$. Thus

$$
\left(M_{\mathbf{t}} \phi\right)(H)=\sum_{\mathbf{h} \in \operatorname{Car}(\mathbf{g})} \int_{\mathbf{h}^{*}} \theta(H ; \lambda)\left|\Pi_{\mathbf{h}}(\lambda)\right|^{2}\left(\int_{G / H} \hat{\phi}_{\mathbf{g}}(g \cdot \lambda) d \dot{g}\right) d \lambda .
$$

Let $D_{\Pi_{t}}$ be the constant coefficient differential operator corresponding to $\Pi_{t}$. Define the distribution $\phi \rightarrow(I \phi)(H)$ by

$$
(I \phi)(H)=D_{\Pi_{\mathbf{t}}}\left[\frac{1}{(2 \pi)^{d}} \Pi_{\mathbf{t}}(H) \int_{G / T} \phi(g \cdot H) d \dot{g}\right],
$$

where $\operatorname{dim} G / T=2 d$. Then $I \phi$ is a canonical distribution on $\mathbf{g}$ which is independent of the choice of positive roots. By a theorem of Harish-Chandra [6], $H \rightarrow(I \phi)(H)$ extends to a continuous function on $\mathbf{t}$ and the $\lim _{H \rightarrow 0}(I \phi)(H)=$ $(-1)^{d} \# W_{K} \phi(0)$.

For $h \in \mathbf{t}$, the Fourier transform of the distribution $\phi \rightarrow(I \phi)(H)$ is given by

$$
(I \phi)(H)=\int_{\mathbf{g}^{*}} V(f ; H) \hat{\phi}(f) d f
$$


where $d f$ is the measure of $\mathbf{g}^{*}$ canonically dual to $d X$, and $V(f ; H)$ is a $G$-invariant bounded function on $\mathbf{g}^{*}$ (see [16]).

Let $\mathbf{h}$ be any Cartan subalgebra of $\mathbf{g}$. Then $V(f ; H)$ is completely determined by its restriction to all the $\mathbf{h}^{*}$. Let $C_{\mathfrak{R}}^{+}=\left\{f \in \mathbf{h}^{*} \mid\left(f, H_{\alpha}\right)>0\right.$, $\left.\alpha \in \Phi_{\mathfrak{R}}^{+}\right\}$. Let $W\left(\mathbf{t}_{\mathbf{C}}, \mathbf{h}_{\mathbf{C}}\right)=\left\{g \in G_{\mathbf{C}} \mid g\left(\mathbf{t}_{\mathbf{C}}\right)=\mathbf{h}_{\mathbf{C}}\right\}$. Then for $f \in C_{\mathfrak{R}}^{+} \subset \mathbf{h}^{*}$, $H \in \mathbf{t}$,

$$
V(f ; H)=\sum_{w \in W\left(\mathbf{t}_{\mathrm{C}}, \mathbf{h}_{\mathbf{C}}\right)} h\left(w ; \Phi_{\mathfrak{R}}^{+} ; H\right) e^{-(f, i w H)},
$$

where the $h\left(w ; \Phi_{\mathfrak{R}}^{+} ; H\right)$ are a set of constants. These constants are uniquely determined by the following properties [7].

3.1. (a) Let $\mathbf{h}=\mathbf{t}$. Then $\Phi_{\mathfrak{R}}^{+}=\{\varnothing\}$, and

$$
h\left(w ; \Phi_{\mathfrak{R}}^{+} ; H\right)= \begin{cases}1, & w \in W_{K}, \\ 0, & w \notin W_{K} .\end{cases}
$$

(b) The $h\left(w ; \Phi_{\mathfrak{R}}^{+} ; H\right)=0$ if $P_{\mathfrak{R}}(i w H) \notin \overline{\sum_{\beta \in \Phi_{\mathfrak{R}}^{+}} \mathfrak{R}^{+} H_{\beta}}$.

(c) Let $\alpha$ be a simple root with respect to the order determined by $\Phi_{\mathfrak{R}}^{+}$. Then $h\left(w ; \boldsymbol{\Phi}_{\mathfrak{R}}^{+} ; H\right)+h\left(s_{\alpha} ; \boldsymbol{\Phi}_{\mathfrak{R}}^{+} ; H\right)=h\left(c_{\alpha} \cdot w ;\left(\boldsymbol{\Phi}_{\mathfrak{R}}^{+}\right)_{\alpha} ; H\right)+h\left(c_{\alpha} \cdot s_{\alpha} \cdot w ;\left(\boldsymbol{\Phi}_{\mathfrak{R}}^{+}\right)_{\alpha} ; H\right)$.

Let $\chi$ be the fixed character of the center $Z$ of $G$. Let $\mathbf{h} \in \operatorname{Car}(\mathbf{g})$. Let $t$ be the maximal compact Cartan subalgebra of $g$. Let $t^{Z}=g^{Z} \cap \mathbf{t}$. Then $\mathbf{t}_{\mathrm{C}}^{Z}=\mathbf{t}^{Z}$.

Define a tempered distribution

$$
\phi \rightarrow \sum_{\gamma \in \mathbf{t}^{2}} \chi(\exp \gamma)(I \phi)(\gamma)
$$

The Fourier transform of this distribution is given by

$$
\sum_{\gamma \in \mathbf{t}^{Z}} \chi(\exp \gamma)(I \Phi)(\gamma)=\int_{\mathbf{g}^{*}} V^{\chi}(f) \hat{\phi}(f) d f,
$$

where $V^{\chi}(f)$ is a $G$-invariant generalized function on $\mathbf{g}^{*}$ supported on the set of regular elements of $\mathbf{g}^{*}$ [16]. Therefore the restriction of $V^{\chi}$ to $\mathbf{h}_{r}^{*}$ is well defined and

$$
\sum_{\gamma \in \mathbf{t}^{Z}} \chi(\exp \gamma)(I \phi)(\gamma)=\sum_{\mathbf{h} \in \operatorname{Car}(\mathbf{g})}\left(\# W_{\mathfrak{R}}\right) \int_{\mathbf{h}_{K}^{*} \oplus C_{\mathfrak{R}}^{+}} V_{\mathbf{h}}^{\chi}(\lambda)|\Pi(\lambda)|(k \phi)(\lambda) d \lambda,
$$

where

$$
V_{\mathbf{h}}^{\chi}(\lambda)=\sum_{\xi \in \mathbf{h}_{\mathbf{C}}^{\mathbf{Z}}} r_{\mathbf{h}}^{\chi}\left(\xi ; \Phi_{\mathfrak{R}}^{+}\right) e^{-i(\lambda, \xi)}, \quad \text { for } \lambda \in C_{\mathfrak{R}}^{+},
$$

and

$$
r_{\mathbf{h}}^{\chi}\left(\xi ; \Phi_{\mathfrak{R}}^{+}\right)=\sum_{w \in W\left(\mathbf{t}_{\mathbf{c}}, \mathbf{h}_{\mathbf{c}}\right)} \chi\left(\exp w^{-1} \xi\right) h\left(w ; \Phi_{\mathfrak{R}}^{+} ; w^{-1} \xi\right) .
$$
ties.

The constants $r_{h}^{\chi}\left(\xi ; \Phi_{\mathfrak{R}}^{+}\right)$are uniquely determined by the following proper- 
3.2. (a) Let $\mathbf{t}$ be the maximal compact Cartan subalgebra. Then

$$
r_{\mathbf{t}}^{\chi}(\gamma)=\chi(\exp \gamma), \quad \gamma \in \mathbf{t}^{Z}
$$

(b) Let $\alpha$ be a simple root of $\Phi_{\mathfrak{R}}^{+}, \xi \in \mathbf{h}_{\mathbf{C}}^{Z}$. Then

$$
r_{\mathbf{h}}^{\chi}\left(\xi ; \Phi_{\mathfrak{R}}^{+}\right)+r_{\mathbf{h}}^{\chi}\left(s_{\alpha} \xi ; \Phi_{\mathfrak{R}}^{+}\right)=r_{\mathbf{h}_{\alpha}}^{\chi}\left(c_{\alpha} \xi ;\left(\Phi_{\mathfrak{R}}^{+}\right)_{\alpha}\right)+r_{\mathbf{h}_{\alpha}}^{\chi}\left(c_{\alpha} s_{\alpha} \xi ;\left(\Phi_{\mathfrak{R}}^{+}\right)_{\alpha}\right) .
$$

(c) The $r_{\mathrm{h}}^{\chi}\left(\xi ; \Phi_{\mathfrak{R}}^{+}\right)=0$ if $i P_{\mathfrak{R}}(\xi) \notin \sum_{\beta \in \Phi_{\mathfrak{R}}^{+}} \mathfrak{R}^{+} H_{\beta}$.

For $\xi \in \mathbf{h}_{\mathbf{C}}^{Z}$ and $\delta \in \mathbf{h}^{Z}, 3.2$ implies that $r_{\mathbf{h}}^{\chi}\left(\xi+\delta ; \Phi_{\mathfrak{R}}^{+}\right)=\chi(\exp \delta) r_{\mathbf{h}}^{\chi}\left(\xi ; \Phi_{\mathfrak{R}}^{+}\right)$. Then $r_{\mathbf{h}}^{\chi}\left(\xi ; \Phi_{\mathfrak{R}}^{+}\right) e^{-i(\xi, \lambda)}$ depends only on the projection of $\xi$ in $\mathbf{h}_{p}$. Let $\xi=$ $\xi_{0}+i \xi_{1}, \xi_{0} \in \mathbf{h}_{k}, i \xi_{1} \in P_{\mathfrak{R}}\left(\mathbf{h}_{\mathbf{C}}^{Z}\right)$. Then

$$
\sum_{\xi \in \mathbf{h}_{\mathrm{C}}^{Z}} r_{\mathbf{h}}^{\chi}\left(\xi ; \Phi_{\mathfrak{R}}^{+}\right) e^{-i(\xi, \lambda)}=\sum_{i \xi_{1} \in P_{\mathfrak{R}}\left(\mathbf{h}_{\mathbf{C}}^{Z}\right)} \sum_{\delta \in \mathbf{h}^{Z}}\left(r_{\mathbf{h}}^{\chi}\left(\xi ; \Phi_{\mathfrak{R}}^{+}\right) e^{-i(\xi, \lambda)}\right) e^{-i(\delta, \lambda)} \chi(\exp \delta)
$$

where $P_{\mathfrak{R}}(\xi)=i \xi_{1}$. Let

$$
R_{\mathbf{h}}^{\chi}(\lambda)=\sum_{\delta \in \mathbf{h}^{Z}} e^{-i(\delta, \lambda)} \chi(\exp \delta)
$$

By the Poisson formula, $R_{\mathrm{h}}^{\chi}(\lambda)$ is a positive generalized function with support on $\mathbf{h}^{*}(\chi)$. By the Weyl integration formula, $R_{\mathbf{h}}^{\chi}(\lambda)$ extends by $G$-invariance to a positive generalized function on $\mathbf{g}^{*}$ with support on $G \cdot\left[\left(\mathbf{h}^{*}(\chi)\right)_{r}\right]$, where $\left[\left(\mathbf{h}^{*}(\chi)\right)_{r}\right]=\left(\mathbf{h}^{*}(\chi) \cap \mathbf{h}_{r}^{*}\right)[16]$

Lemma 3.3. Let $\chi$ be the character of the center $Z$ of $G$. Let $m^{\chi}\left(\xi ; \Phi_{\mathfrak{R}}^{+}\right)$be the constants defined by (2.6). Then

$$
m^{\chi}\left(\xi ; \Phi_{\mathfrak{R}}^{+}\right)=r_{\mathbf{h}}^{\chi}\left(2 \pi i \xi ; \Phi_{\mathfrak{R}}^{+}\right), \quad \xi \in L_{Z}
$$

Proof. By Proposition 2.4 and the unicity of the constants, equality is established.

Let $\xi \in \mathbf{h}_{\mathbf{C}}^{Z}, \xi=\xi_{0}+i \xi_{1}, \xi_{0} \in \mathbf{h}_{k}, i \xi_{1} \in P_{\mathfrak{R}}\left(\mathbf{h}_{\mathbf{C}}^{Z}\right), \mu \in \mathbf{h}_{k}^{*}(\chi)$. Define

$$
r_{\mathbf{h}}^{\chi}\left(\xi_{1} ; \Phi_{\mathfrak{R}}^{+}\right)=r_{\mathbf{h}}^{\chi}\left(\xi ; \Phi_{\mathfrak{R}}^{+}\right) e^{i(\xi, \mu)}
$$

Let $P(b, \nu)$ be the Plancherel product defined by (2.3).

Theorem 3.4. Let $G$ be the universal covering group with Lie algebra $\mathbf{g}=$ $\operatorname{so}(p, q ; \mathfrak{R}), p+q=2 n+1, p<q$, and $p, q \neq 0,1,2$. Let $\chi$ be the character of the center $Z$ of $G$. Let $\mu \in \mathbf{h}_{k}^{*}(\chi), \xi \in L_{Z}, \xi=i \xi_{0}+\xi_{1}, \xi_{0} \in \mathbf{h}_{k}$, $i \xi_{1} \in P_{\mathfrak{R}}\left(\mathbf{h}_{\mathbf{C}}^{Z}\right), \nu \in \mathbf{h}_{p}^{*}$. Then

$$
\frac{1}{\left[H_{K}: H_{K}^{0} Z\right]} \sum_{\substack{b \in \hat{H}_{K}(\chi) \\ \log b=i \mu}}(\operatorname{dim} b)^{2} P(b, \nu)=\sum_{2 \pi \xi_{1} \in P_{\mathfrak{R}}\left(\mathrm{h}_{\mathrm{C}} \mathrm{z}\right)} r_{\mathbf{h}}^{\chi}\left(2 \pi i \xi_{1} ; \Phi_{\mathfrak{R}}^{+}\right) .
$$


Proof. By (2.5)

$$
P(b, \nu)=\sum_{\xi_{1} \in \Lambda\left(\Sigma_{\mathfrak{R}}\right)} \tilde{\chi}\left(\xi_{1}\right) d\left(\xi_{1} ; \Sigma_{\mathfrak{R}}^{+}\right) e^{-\left(2 \pi \nu, \xi_{1}\right)}
$$

Then

$$
\frac{1}{\left[H_{K}: H_{K}^{0} Z\right]} \sum_{\substack{b \in \hat{H}_{K}(\chi) \\ \log b=i \mu}}(\operatorname{dim} b)^{2} P(b, \nu)=\sum_{\xi_{1} \in P_{\mathfrak{R}}\left(L_{Z}\right)} \tilde{\chi}\left(\xi_{1}\right) d\left(\xi_{1} ; \Sigma_{\mathfrak{R}}^{+}\right) e^{-2 \pi\left(\xi_{1}, \nu\right)}
$$

follows as a consequence of the first statement of Lemma 2.3.

When $\mu \in \mathbf{h}_{k}^{*}(\chi), \xi \in L_{Z}, \xi=i \xi_{0}+\xi_{1}$, (3.6) becomes

$$
\begin{aligned}
r_{\mathbf{h}}^{\chi}\left(2 \pi i \xi_{1} ; \Phi_{\mathfrak{R}}^{+}\right) & =r_{\mathbf{h}}^{\chi}\left(2 \pi i \xi ; \Phi_{\mathfrak{R}}^{+}\right) e^{i(2 \pi i \xi, \mu)} \\
& =m^{\chi}\left(\xi ; \Phi_{\mathfrak{R}}^{+}\right) e^{-i 2 \pi\left(\xi_{0}, \mu\right)} e^{-2 \pi\left(\xi_{1}, \mu\right)} \\
& =\tilde{\chi}\left(\xi_{1}\right) d\left(\xi_{1} ; \Sigma_{\mathfrak{R}}^{+}\right) e^{-2 \pi\left(\xi_{1}, \mu\right)}
\end{aligned}
$$

where the last statement follows from Lemma 3.3 and (2.6). Thus

$$
\begin{aligned}
\sum_{2 \pi \xi_{1} \in P_{\mathfrak{R}}\left(\mathrm{h}_{\mathrm{C}}^{Z}\right)} r_{\mathbf{h}}^{\chi}\left(2 \pi i \xi_{1} ; \Phi_{\mathfrak{R}}^{+}\right) & =\sum_{\xi_{1} \in P_{\mathfrak{R}}\left(L_{Z}\right)} \tilde{\chi}\left(\xi_{1}\right) d\left(\xi_{1} ; \Sigma_{\mathfrak{R}}^{+}\right) e^{-\left(2 \pi \xi_{1}, \nu\right)} \\
& =\frac{1}{\left[H_{K}: H_{K}^{0} Z\right]} \sum_{\substack{b \in \hat{H}_{K}(\chi) \\
\log b=i \mu}}(\operatorname{dim} b)^{2} P(b, \nu),
\end{aligned}
$$

proving the theorem.

Let $\lambda \in \mathbf{h}^{*}(\chi)$. Define

$$
P_{\mathrm{h}}^{\chi}(\lambda)=\frac{1}{\left[H_{K}: H_{K}^{0} Z\right]} \sum_{\substack{b \in \hat{H}_{K}(\chi) \\ \log b=i \mu}}(\operatorname{dim} b)^{2} P(b, \nu)
$$

Let $V_{\mathrm{h}}^{\chi}(\lambda)$ be the $G$-invariant function on $\mathrm{g}^{*}$ given by (3.3). Let $R_{\mathrm{h}}^{\chi}(\lambda)$ be the $G$-invariant positive generalized function on $\mathbf{g}^{*}$ with support on $G \cdot\left[\left(\mathbf{h}^{*}(\chi)\right)_{r}\right]$, defined by (3.5).

Theorem 3.5. Let $G$ be the universal covering group with Lie algebra $\mathbf{g}=$ so $(p, q ; \mathfrak{R}), p+q=2 n+1, p<q$, and $p, q \neq 0,1,2$. Let $\chi$ be the character of the center $Z$ of $G$. Let $\lambda \in \mathbf{h}^{*}(\chi)$. Then

Proof. Apply Theorem 3.4 to (3.4). Q.E.D.

$$
V_{\mathrm{h}}^{\chi}(\lambda)=R_{\mathrm{h}}^{\chi}(\lambda) P_{\mathrm{h}}^{\chi}(\lambda)
$$

Recall the character $\chi$ was defined such that $\chi\left(\varepsilon_{p} \cdot \varepsilon_{q}\right)=-1, \varepsilon_{p} \cdot \varepsilon_{q} \in Z$. If $\chi$ is any character on $Z$ such that $\chi\left(\varepsilon_{p} \cdot \varepsilon_{q}\right)=1$, then Theorem 3.5 was proved by Vergne [16].

The Theorem 3.5 is a precise version of the Poisson-Plancherel conjecture after summing over all the $\chi \in \hat{Z}_{G}$, in terms of positive generalized functions concentrated on $\mathbf{g}_{G}^{*}$. 


\section{REFERENCES}

1. M. Andler, La formule de Plancherel pour les groupes algébriques complexes unimodulaires, Acta Math. 154 (1985).

2. J.-Y. Charbonnel, La formule de Plancherel pour un groupe résoluble connexe. II, Math. Ann. 250 (1980), 1-34.

3. J. Dixmier, Les $C^{*}$-algèbres et leurs répresentations, Gauthier-Villars, Paris, 1964.

4. M. Duflo, Représentations unitares des groupes de Lie et méthode des orbits, G.M.E.L., Bordas, Paris, 1982.

5. __ On the Plancherel formula for almost algebraic real Lie groups, Lecture Notes in Math., vol. 1077, Springer, 1984.

6. Harish-Chandra, Some results on an invariant integral on a semi-simple Lie algebra, Ann. of Math. (2) 80 (1964), 551-593.

7. __ Discrete series for semi-simple Lie groups. I, Acta Math. 113 (1965), 241-318.

8. __ Harmonic analysis on real reductive groups. III. The Maass-Selberg relations and the Plancherel formula, Ann. of Math. (2) 104 (1976), 117-201.

9. R. Herb, Character formulas for discrete series on semisimple Lie groups, Nagoya Math. J. 64 (1976), 47-61.

10. R. Herb and J. A. Wolf, The Plancherel theorem for general semisimple groups, Compositio Math. 57 (1986), 271-355.

11. A. A. Kirillov, Characters of unitary representations of Lie groups, Functional Anal. Appl. 2 (1968), 133-146.

12. D. Peterson and M. Vergne, Recurrence relations for Plancherel functions, Lecture Notes in Math., vol. 1243, Springer, 1987.

13. W. Rossman, Kirillov's character formula for reductive Lie groups, Invent. Math. 48 (1978), 207-220.

14. M. Sugiura, Conjugate classes of Cartan subalgebras in real semisimple Lie algebras, J. Math. Soc. Japan 2 (1959), 374-434.

15. P. Torasso, La formule de Poisson-Plancherel pour un groupe de Takiff associé à un groupe de Lie semi-simple à centre fini, preprint (1984).

16. M. Vergne, A Poisson-Plancherel formula for semi-simple Lie groups, Ann. of Math. (2) 115 (1982), 639-666.

Department of Physics, Massachusetts Institute of Technology, Cambridge, MasSACHUSETTS 02139 